\title{
The internal representation of temporal orienting: A temporal pulse-accumulation and attentional-gating-based account
}

\author{
Xiaorong Cheng ${ }^{1,2,3} \cdot$ Yu Mao ${ }^{1,2,3} \cdot$ Yang Lei ${ }^{1,2,3} \cdot$ Chunyan Lin ${ }^{1,2,3} \cdot$ Chunmiao Lou ${ }^{1,2,3} \cdot$ Zhao Fan $^{1,2,3}$. \\ Xianfeng Ding ${ }^{1,2,3}$
}

Accepted: 8 October 2020 / Published online: 23 November 2020

(C) The Psychonomic Society, Inc. 2020

\begin{abstract}
Timing can be processed explicitly or implicitly. Temporal orienting is a typical implicit timing through which we can anticipate and prepare an optimized response to forthcoming events. It is, however, not yet clear whether mechanisms such as temporalpulse accumulation and attentional gating (more attention, more accumulated temporal pulses) underly the internal representations of temporal orienting, as in explicit timing. To clarify this, a dual-task paradigm, consisting of a temporal orienting and an interference task, was adopted. Consistent with the temporal-pulse-accumulation and attentional-gating model, reaction times to the target detection of temporal orienting increased as the interference stimuli were temporally closer to the target, i.e., a location effect for temporal orienting. This effect is likely due to attention being diverted away from temporal orienting to monitor the occurrence of the interference stimuli for a longer time, resulting in greater temporal pulse loss and less accurate temporal orienting for conditions with later interference stimuli. The temporal-pulse-accumulation aspect in temporal orienting received further support by taking an explicit duration reproduction (containing a second temporal-pulse accumulation) as the interference task. On the one hand, temporal orienting became less accurate with increased temporal-pulse-accumulation overlaps between the dual tasks; on the other hand, two-way (one for temporal orienting and the other for duration reproduction), rather than oneway, location effects were observed, implying processing conflicts between the two temporal-pulse accumulations. Taken together, these results suggest that implicit and explicit timing may share common mechanisms upon internal temporal representations.
\end{abstract}

Keywords Implicit and explicit timing $\cdot$ Temporal orienting $\cdot$ Temporal pulse accumulation $\cdot$ Attentional gating $\cdot$ Location effect

\section{Introduction}

Subjective temporal processing can be divided into explicit and implicit timing (Coull \& Nobre, 2008; Praamstra, Kourtis, Kwok, \& Oostenveld, 2006; Zelaznik, Spencer, \& Ivry, 2002). The essential difference between them lies in

Xiaorong Cheng, Yu Mao and Yang Lei contributed equally to this work.

Zhao Fan

z.fan@mail.ccnu.edu.cn

$\triangle$ Xianfeng Ding

xianfengding@mail.ccnu.edu.cn

1 Key Laboratory of Adolescent Cyberpsychology and Behavior (CCNU), Ministry of Education, Wuhan 430079, China

2 School of Psychology, Central China Normal University (CCNU), Wuhan 430079, China

3 Key Laboratory of Human Development and Mental Health of Hubei Province, Wuhan 430079, China whether clear and direct time estimates are required. In an explicit timing, participants were asked to make direct time estimates, whereas no such task components at the conscious level were included in an implicit timing. Instead, participants were instructed to complete a non-temporal task with its performance facilitated by either an endogenous temporal cue or a background time structure (Nobre \& van Ede, 2018; Piras \& Coull, 2011).

Temporal orienting is a typical implicit timing where the response speed (Coull \& Nobre, 1998; Nobre \& Rohenkohl, 2014) of a non-temporal task is modulated by endogenous guidance of attention along a temporal dimension. In a classical temporal-orienting task (Coull \& Nobre, 1998), participants were required to detect a target as soon as possible. An informative, symbolic cue was used intentionally to predict with high probability the moment at which the target would occur, i.e., a short or a long interval after the cue offset. Reaction times (RTs) in the target detection task were reduced by valid relative to invalid cues, suggesting that attention was 
directed into specific moments by the valid cues, i.e., a temporal orienting (or a "cue validity") effect.

Concerning the internal representation of explicit timing, the dominant account is the so-called attentional-gate model (Zakay \& Block, 1997), which relies on temporal-pulse accumulation and attentional gating (more attention, more accumulated temporal pulses). According to this model, attentional resource is necessary for the operation of an attentional gate between a pacemaker that generates "subjective time units" (Zakay \& Block, 1997) or "temporal pulses" (Creelman, 1962; Gibbon, Church, \& Meck, 1984; Treisman, 1963) for the target interval and an accumulator that stores transferred temporal pulses. The final count of the accumulated temporal pulses in the accumulator decides the subjective duration of the target interval. The opening and closing of the gate heavily depend on the amount of allocated attention. The "temporalpulse-accumulation and attentional-gating" nature of explicit timing received consistent support by a phenomenon dubbed the "location effect" (Fortin \& Tremblay, 2006) that exists in various explicit-timing tasks (Buffardi, 1971; Casini \& Macar, 1997; Fortin, Bédard, \& Champagne, 2005; Gaudreault, Fortin, \& Macar, 2010), including dual-task interference paradigms (Gaudreault et al., 2010) and break paradigms in interval timing (Fortin \& Massé, 2000). For example, in a typical dual-task interference paradigm, participants were required to discriminate whether a target interval was short or long and at the same time to judge the strength of a sudden event, composed of enhanced intensity, occurring at any time during the target interval. It was found that the perceived duration of the target interval was modulated by the location of the non-temporal-interference task (Casini \& Macar, 1997). The interval was judged as being shorter when the concurrent interference event occurred later within the target interval, i.e., a typical location effect for explicit timing. Such an effect, particularly the linear relationship between performance of an explicit timing and location levels of a concurrent interference task, is consistent with the prediction of the attentionalgate model (Casini \& Macar, 1997) within the context of attention sharing (i.e., attentional resource is limited and must be allocated and shared between temporal processing and other interference tasks; Fortin \& Massé, 2000; Macar, Grondin, $\&$ Casini, 1994). Since the opening and closing of the gate rely on the allocated attention, the attentional monitoring to the occurrence of the interference stimuli would disturb the attentional allocation to the explicit timing and its temporal-pulse accumulation. For later interference stimuli, attentional deviation from the temporal processing lasts for a longer time, resulting in larger diminution of the accumulated temporal pulses and eventually a shorter perceived duration. Thus, the location effect provided consistent evidence that the internal representation of explicit timing involves mechanisms of temporal-pulse accumulation and attentional gating (Casini \& Macar, 1997).
Whether implicit timing, such as temporal orienting, shares similar mechanisms to explicit timing is still under debate. Some researchers believe that both implicit and explicit timing involve similar temporal-pulse accumulation and attentional gating (Merchant, Zarco, Bartolo, \& Prado, 2008; Spencer \& Zelaznik, 2003) and all obey psychophysical laws, including the psychophysical dependence between subjective time and real time, and Weber's law, i.e., the scalar variability (Piras \& Coull, 2011). One study (Casini, Ramdani-Beauvir, Burle, \& Vidal, 2013) found that one night of sleep deprivation produced the same pattern of effects for explicit timing in the suprasecond range and for implicit timing in the millisecond range, implying a common mechanism between suprasecond explicit timing and subsecond implicit timing.

However, other studies (Coull, Davranche, Nazarian, \& Vidal, 2013; Coull \& Nobre, 2008; Zelaznik et al., 2002) have suggested that these two types of timing are essentially different and could not be explained by a unified model with the same temporal representations. This idea was based on some recent findings demonstrating that explicit and implicit timing activated different brain regions (Coull et al., 2013; Coull \& Nobre, 2008), possessed distinct developmental trajectories (Droit-Volet \& Coull, 2016), and showed dissociable performance on both motor and perceptual timing paradigms (Zelaznik et al., 2002) and oculomotor control tasks (Ameqrane, Pouget, Wattiez, Carpenter, \& Missal, 2014). The dissociations between implicit and explicit timing might suggest different ontogenetic origins for them. This may lead to distinct developmental trajectories (Droit-Volet \& Coull, 2016) and different internal temporal representations between them (Ameqrane et al., 2014; Zelaznik et al., 2002).

Thus, it is yet to be clarified whether a temporal-pulse accumulation and attentional gating-based framework, originally stemming from explicit timing, also underlies implicit timing, for example, temporal orienting. If so, we would observe a similar location effect for temporal orienting in similar dual-task interference paradigms, namely a slower target detection (greater temporal pulse loss leads to less accurate temporal orienting) following the later occurrence of the interference stimuli, which is a critical signature for a temporal-pulse accumulation and attentional gating-based timing model. In addition, since the location effect is largely due to a reallocation of controlled attention to monitor the occurrence of an interference signal, we would expect two specific predictions in a dual-task/stimuli paradigm. First, the location effect for temporal orienting should be absent or substantially reduced when participants are required to ignore the interference task, due to the fact that no controlled attentional shifts but only automatic shifts of attention (i.e., attention is captured by the onset of the interference stimuli in an automatic way) are involved now. Second, for an interference task that is more difficult with a higher processing demand, such 
as a pitch discrimination with shorter tones, more controlled attention would be allocated to monitor the interference tones (Fortin \& Massé, 2000; Macar et al., 1994), which results in a larger location effect eventually. Therefore, we would expect that the magnitude of the location effect for temporal orienting is modulated by the processing demand (task difficulty) of the interference task. Similar effects have been observed for explicit timing (Champagne \& Fortin, 2008; see Experiment 3 for more details). It is thus intriguing to ask further whether such effects exist in temporal orienting.

Moreover, whether the internal representation of temporal orienting possesses a mechanism based on temporal-pulse accumulation and attentional gating, i.e., similar to that of explicit timing, can be investigated further by directly pitting temporal orienting with an explicit timing. Suppose in a dual-task paradigm, an explicit duration reproduction is taken as the interference task, which introduces an extra temporalpulse-accumulation component into the dual tasks. This would elicit additional processing conflicts between the two temporal-pulse accumulations relative to conditions when a non-temporal-interference task is used. Indeed, similar conflicts of temporal-pulse accumulation, though between multiple explicit timings rather than between an explicit and an implicit timing, have been observed in recent literature (Ayhan, Revina, Bruno, \& Johnston, 2012; Cheng, Yang, Han, Ding, \& Fan, 2014; van Rijn \& Taatgen, 2008).

On the basis of the temporal-pulse-accumulation conflicts, two potential outcomes might be expected. First, the temporal orienting might become less accurate with increased temporalpulse-accumulation overlaps (longer target durations in the temporal-interference task would produce larger temporalpulse-accumulation overlaps between the dual tasks). Second, when the interference stimuli are temporally closer to the temporal orienting target (i.e., at later locations), the temporal-pulse-accumulation conflicts between the temporal orienting and the duration encoding of the to-be-reproduced interference stimuli become more severe since more attention is diverted to monitor the occurrence of the temporal-orienting target (which becomes more imminent now) and less attention is available to coordinate the temporal-pulse-accumulation conflicts. The increased temporal-pulse-accumulation conflicts at later locations (note, the duration of the temporalpulse-accumulation conflicts is subject to the duration of the to-be-reproduced interference stimuli and thus not modulated by location levels) would result in fewer accumulated temporal pulses for the interference stimuli eventually. Thus, the location effect for the interference task is likely to emerge in conditions involving the temporal-pulse-accumulation conflicts, leading to two-way (one for temporal orienting and the other for duration reproduction), rather than one-way, location effects in these conditions. On the contrary, if the location effect for temporal orienting is induced by a simple processing interference between the two tasks with no specific connection to the temporal-pulse accumulation and attentional gating mechanism, i.e., "a simple interference hypothesis," we would expect to observe a wide occurrence of the location effect for the interference task, regardless of using a temporal (containing an extra temporal-pulse accumulation and thus resultant temporal-pulse-accumulation conflicts) or a non-temporal (containing no extra temporal-pulse accumulation and thus no corresponding conflicts) interference task.

Besides the "simple interference hypothesis," another alternative hypothesis accounting for the location effect for temporal orienting is related to the well-established variableforeperiod (FP) effect (Woodrow, 1914), in which the performance to an imperative stimulus (S2, e.g., the stimuli of the interference task) was improved with an increase of the FP that was defined as the interval between the offset of a warning stimulus (S1, e.g., the cues in the temporal-orienting task) and the onset of the S2. The variable-FP effect was explained as the recruitment of preparatory (Niemi \& Näätänen, 1981) or attentional (Grondin \& Rammsayer, 2003) resources increasing in keeping with the conditional probability of the occurrence of the S2 with the elapse of time, i.e., the Hazard function (Bertelson \& Tisseyre, 1968). An alternative FPeffect-based account for the location effect for temporal orienting could be that more preparatory or attentional resources are diverted away from the temporal orienting due to the interference stimuli-related FP effect, i.e., "a foreperiod-effect-based hypothesis." Note that, similar to the "simple interference hypothesis," this account also does not rely on a temporal-pulse accumulation and attentionalgating mechanism for the internal representation of temporal orienting. Instead, the RT increase at the later locations is only a by-product of the resource reallocation at different location levels. According to this hypothesis, the increase of allocated resource (and the resultant performance changes) to the interference stimuli at later location levels should occur together with the location effect for temporal orienting. These two alternative hypotheses for the potential location effect for temporal orienting require further investigation.

\section{The present study}

To the best of our knowledge, no study has yet tackled the issue of whether a temporal-pulse accumulation and attentional-gating mechanism underlie the internal representation of temporal orienting by directly testing a potential location effect for temporal orienting and its characteristics. Therefore, we explored the nature of the processes involved in temporal orienting with six experiments (Experiments 1a, $1 \mathrm{~b}$, and 2-5). Experiments $1 \mathrm{a}$ and $1 \mathrm{~b}$ aimed to explore 
whether there was a location effect for temporal orienting. These two experiments differed on the orders of single- and dual-task blocks only (see Experiments $1 \mathrm{a}$ and $1 \mathrm{~b}$ for more details). Experiment 2 further evaluated to what extent the location effect relied on automatic shifts of attention. Experiment 3 aimed to explore whether the magnitude of the location effect is modulated by the processing demand (task difficulty) of the non-temporal-interference task. Two more experiments (Experiments 4 and 5) with stricter experimental controls were carried out to verify our explanation of the location effect and to rule out several alternative explanations by adopting a non-temporal task and an explicittiming task as the interference task, respectively. Specifically, Experiment 4 was conducted to assess the stability of the location effect in the context of matched location levels with equal spacing for the short and long cuetarget intervals and to evaluate the possibility of a psychological refractory period (PRP)-based alternative interpretation. Experiment 5 further tested the role of temporalpulse accumulation in temporal orienting by directly pitting a temporal-orienting task with an explicit duration reproduction task. Additional cross-experimental comparisons not only investigated further the temporal-pulseaccumulation nature of the internal representation of temporal orienting, but also explored other alternative hypotheses for the location effect for temporal orienting, including "the simple interference hypothesis" and "the FP effectbased hypothesis."

\section{General methods and results overview}

\section{Participants and procedures}

The present study was approved by the Ethics Committee of the Central China Normal University (CCNU). All participants gave their informed consent to participate. Prior to each experiment, we conducted an a priori sample size calculation (GPower 3.1; Faul, Erdfelder, \& Lang, 2007). Assuming an alpha of 0.05 and power of 0.8 , a minimum sample size needed to detect a medium effect size ( $f=0.25$ ) with sufficient power was 19 for Experiments 1-5 (ANOVA, repeated measures, within factors, and six measurements). The above criterion was met in the present study, leading to a recruitment of 20 participants in Experiment 1a (13 females and seven males; mean age 20.7 years), Experiment $1 \mathrm{~b}$ (13 females and seven males; mean age 20.9 years) and Experiment 2 (11 females and nine males; mean age 19.8 years), 23 participants in Experiment 3 (13 females and ten males; mean age 20.1 years), and 28 participants in Experiment 4 ( 22 females and six males; mean age 20.0 years) and Experiment 5 ( 22 females and six males; mean age 20.8 years). All participants were paid for their participation. They were right-handed, with normal or corrected-to-normal vision, and were naive to the purpose of the study.

The experiments took place in a quiet room. E-prime 2.0 (Psychology Software Tools, Inc.) was used to generate stimuli and run all the experiments. Stimuli were presented on a 17 -in. CRT monitor with a $1,024 \times 768$ resolution at a refresh rate of $100 \mathrm{~Hz}$. Participants' responses were recorded via a keyboard connected to the PC with their head stabilized by a chin rest and a viewing distance of $57 \mathrm{~cm}$.

\section{Basic dual-task/stimuli paradigm: Stimuli}

All six experiments included a basic dual-task/stimuli paradigm. The stimuli and procedure in Experiment 1a (see Fig. 1) are presented here to explain the basic paradigm. Experiment 1a consisted of trials with a single task and those with a dual task. The single task was a temporal-orienting task and the dual task consisted of a temporal-orienting task and a pitch discrimination task (see Fig. 1).

In the temporal-orienting task, all stimuli were presented in the display centre against a black background. Each trial began with the presentation of a red "+" fixation for a random duration between $500 \mathrm{~ms}$ and $1,000 \mathrm{~ms}$. A temporal cue, i.e., a short $\left(3.4^{\circ} \times 1.3^{\circ}\right)$ or a long $\left(7.5^{\circ} \times 1.3^{\circ}\right)$ red bar, was then presented for $750 \mathrm{~ms}$. The short bar and the long bar indicated that a target, i.e., a white circle with a radius of $3.0^{\circ}$ and a duration of $100 \mathrm{~ms}$, would appear at $2,000 \mathrm{~ms}$ and 3,500 ms, respectively, after the offset of the cue. In other words, for the cue-valid condition that included $75 \%$ of all trials, a short bar preceded a relatively short interval of $2,000 \mathrm{~ms}$, and a long bar preceded a relatively long interval of $3,500 \mathrm{~ms}$. In the other $25 \%$ trials of the cue-invalid condition, a short bar preceded a 3,500 -ms interval and a long bar preceded a 2,000-ms interval. Participants were explicitly informed that the temporal cue was highly predictive with regard to the moment of the target onset and were required to indicate the target onset by pressing the space bar as quickly as possible. Following the response to the target, or after $1,100 \mathrm{~ms}$ from the offset of the target in case of a missed response, the next trial was elicited.

The pitch discrimination task required participants to classify a tone as being high-pitch $(50 \mathrm{~dB}, 1,020 \mathrm{~Hz})$ or low-pitch $(50 \mathrm{~dB}, 985 \mathrm{~Hz})$ by pressing either the "J" or the "F" key. The auditory stimuli were created with the GoldWave software (GoldWave, Inc.). Participants received auditory stimulation binaurally through a headphone (MDR-ZX300, Sony, Inc.). The mappings between the pitch tones and the two response keys were counterbalanced across participants. The tone, lasting for $50 \mathrm{~ms}$, appeared within a critical time window that covered $400-1,000 \mathrm{~ms}$ from the start of the $2,000 \mathrm{~ms}$ interval or $500-2500 \mathrm{~ms}$ from the start of the $3,500-\mathrm{ms}$ interval, 


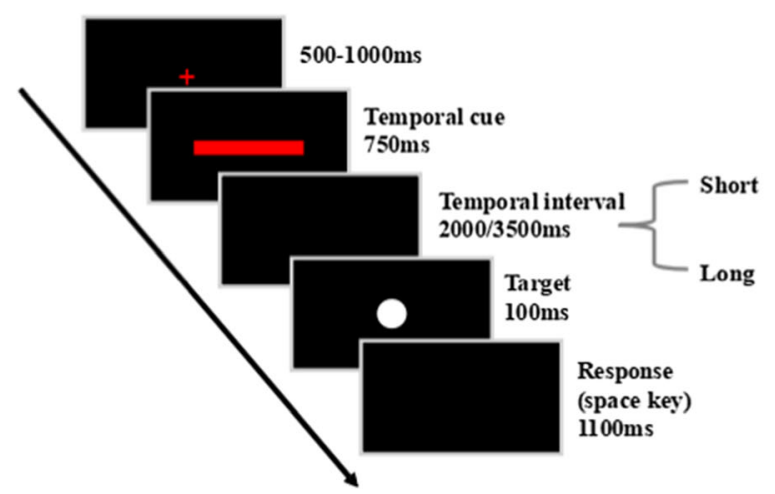

Fig. 1 Schematic representation of events for a dual-task trial in Experiments $1 \mathrm{a}$ and $1 \mathrm{~b}$. The three location levels (early, middle, and late), corresponding to the three interval-sections, are shown and filled in yellow for the 2,000-ms interval and 3,500-ms interval conditions,

respectively. The absence of the tone before and after the critical time windows was designed to minimize potential low-level backward- and forward-masking of the tone to the preceding temporal cue and the subsequent target. The location of the tone had three levels to accommodate the early, middle, and late occurrence of the pitch discrimination task. Accordingly, the critical time window was divided into three interval sections, i.e., $400-600 \mathrm{~ms}, 600-800 \mathrm{~ms}$, and 800 $1,000 \mathrm{~ms}$ for the 2,000-ms interval conditions; or $500-1,150$ $\mathrm{ms}, 1150-1,850 \mathrm{~ms}$, and $1,850-2,500 \mathrm{~ms}$ for the 3,500-ms interval conditions, corresponding to the early, middle, and late locations of the tones, respectively. The criteria for the section division were as follows: for the 2,000-ms interval, the total time window of $600 \mathrm{~ms}(1,000 \mathrm{~ms}$ minus $400 \mathrm{~ms}$, equal to 12 tone durations of $50 \mathrm{~ms}$; see Fig. 1) was divided equally into three $200-\mathrm{ms}$ (equal to four tone durations) interval sections; for the 3,500-ms interval, the total time window of $2,000 \mathrm{~ms}$ ( $2,500 \mathrm{~ms}$ minus $500 \mathrm{~ms}$, equal to 40 tone durations; see Fig. 1) was divided equally into three 650 -ms (equal to 13 tone durations) interval-sections and a remaining $50 \mathrm{~ms}$ (equal to one tone duration) that was appended into the middle interval section to keep the early and the late interval sections exactly the same. The tone could appear randomly at any time point within each of the three interval sections with equal possibilities. Thus, the mean time points of the three interval sections were $500 \mathrm{~ms}, 700 \mathrm{~ms}$, and $900 \mathrm{~ms}$ for the 2,000-ms interval, or $825 \mathrm{~ms}, 1,500 \mathrm{~ms}$, and $2,175 \mathrm{~ms}$ for the $3,500-\mathrm{ms}$ interval. Considering the fact that the tones only appeared within the first $1,000 \mathrm{~ms}$ of the $2,000 \mathrm{~ms}$ interval or within the first $2,500 \mathrm{~ms}$ of the 3,500-ms interval, the mean time points of the three interval sections can be converted into percent location levels by dividing $1,000 \mathrm{~ms}$ and $2,500 \mathrm{~ms}$, respectively, i.e., $50 \%, 70 \%$, and $90 \%$ for the $2,000-\mathrm{ms}$ interval or $33 \%$, $60 \%$, and $87 \%$ for the $3,500-\mathrm{ms}$ interval. Participants were instructed to give their $\mathrm{F} / \mathrm{J}$ key-pressing response to the pitchdiscrimination task as soon as they heard the pitch and a failure to do so before the onset of the target would nullify the trial. For

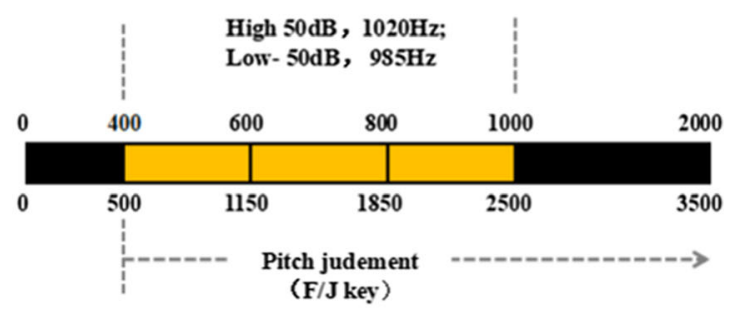

respectively. The tone for the pitch discrimination was present randomly at any time within each interval-section with equal possibilities. The tones were absent in the single-task trials in Experiments $1 \mathrm{a}$ and $1 \mathrm{~b}$

a dual-task trial, a response to the target, i.e., space-bar pressing, should always be given after completing a response to the pitch discrimination, i.e., $\mathrm{F} / \mathrm{J}$ key pressing.

\section{Basic dual-task/stimuli paradigm: Procedure}

The data collection for each participant took two consecutive days. The single-task condition was tested on the first day. Before the formal test, a training session, consisting of 36 trials of the cue-valid condition, was provided for participants to learn the cue-temporal interval contingency. After the training session, participants completed three blocks of 144 trials in total. Each block consisted of 48 trials, including 36 cue-valid and 12 cue-invalid trials, resulting in a $75 \%$ cue validity.

On the next day, participants performed the dual-task condition. Before the formal test, participants had to complete two training sessions, one for the pitch discrimination task and the other for the dual tasks. After the training, participants completed eight dual-task blocks of 384 trials in total. Each block consisted of 48 trials, including 36 cue-valid and 12 cueinvalid trials with a cue validity of $75 \%$. The pitch level, the interval level, and the location level were fully crossed for both the cue-valid and the cue-invalid trials, and the different types of trials were randomly intermixed within each single/dual-task block. Thus, the temporal expectancy driven by symbolic cues was manipulated on a trial-by-trial basis. The single-task blocks took about $15 \mathrm{~min}$ and the dual-task blocks took about $40 \mathrm{~min}$. No feedback was provided in the formal test.

\section{Basic dual-task/stimuli paradigm: Data analysis}

To analyze the location effect, two within-subject variables, i.e., Interval (short, long) and Tone location (early, middle and 
Table 1 Stimulus and instruction/task differences among the dual-task-stimuli (i.e., a white circle target and an interference tone) paradigms in all experiments (see text for more details)

\begin{tabular}{|c|c|c|c|c|c|}
\hline Experiment & $\begin{array}{l}\text { Instruction/Task } \\
\text { to circles }\end{array}$ & $\begin{array}{l}\text { Instruction/Task } \\
\text { to tones }\end{array}$ & Tone location levels & Tone duration(s) & Tone frequency(ies) \\
\hline $1 \mathrm{a}$ and $1 \mathrm{~b}^{\wedge}$ & Temporal orienting ${ }^{*}$ & Pitch discrimination $^{*}$ & $50 / 33,70 / 60$ and $90 / 87 \%$ for short/long intervals & $50 \mathrm{~ms}$ & $1,020 / 985 \mathrm{~Hz}$ \\
\hline 2 & Temporal orienting* & Tone-ignored & $50 / 33,70 / 60$ and $90 / 87 \%$ for short/long intervals & $50 \mathrm{~ms}$ & $1,020 / 985 \mathrm{~Hz}$ \\
\hline 3 & Temporal orienting* & Pitch discrimination $^{*}$ & $50 / 33,70 / 60$ and $90 / 87 \%$ for short/long intervals & $50 / 100 \mathrm{~ms}$ & $1,020 / 985 \mathrm{~Hz}$ \\
\hline 4 & Temporal orienting* & Pitch discrimination ${ }^{\#}$ & $25 / 25,50 / 50$ and $75 / 75 \%$ for short/long intervals & $50 \mathrm{~ms}$ & $1,020 / 985 \mathrm{~Hz}$ \\
\hline 5 & Temporal orienting ${ }^{*}$ & Duration reproduction ${ }^{\#}$ & $25 / 25,50 / 50$ and $75 / 75 \%$ for short/long intervals & $\begin{array}{c}300 / 400 \mathrm{~ms} \text { or } \\
525 / 700 \mathrm{~ms}\end{array}$ & $1,000 \mathrm{~Hz}$ \\
\hline
\end{tabular}

\footnotetext{
Experiments $1 \mathrm{a}$ and $1 \mathrm{~b}$ only differed on the orders of single- and dual-task blocks

* Speeded response to the onset of a stimulus

\# Unspeeded response given at the end of a trial
}

late), were adopted as independent variables. To analyze the temporal orienting effect, three within-subject variables were taken as independent variables, including Interval (short, long), Cue validity (valid, invalid), and Task (single task, dual task). Participants' RT to the target detection was the dependent variable for the above analyses. The slope of functions relating Tone location to RT (Gaudreault \& Fortin, 2013), representing the speed of temporal-pulse accumulation (Fortin, 2003), was also used in the location effect analysis. Similar to Gaudreault and Fortin (2013), the location levels (early, middle, and late) were transformed into corresponding quantitative percentage values $(50,70$, and 90 for the 2,000ms interval and 33,60, and 87 for the 3,500-ms interval, respectively) first. Then, linear regressions using the location percentage values to predict RTs were performed to calculate the slopes of the linear functions relating Tone location to RT for each participant at a particular condition. A steeper slope indicated a larger location effect (Fortin, 2003). Effect sizes were also provided for critical analysis results. More specifically, Cohen's d (Cohen, 1988) was calculated and reported for all significant contrasts, and $\eta_{p}^{2}$ (Kline, 2004) was measured and reported for all the repeated-measures ANOVA ${ }^{1}$ analyses.

\section{Summarized designs for all the experiments}

The paradigms and tasks used in the six experiments are summarized in Table 1 . These experiments differed in aspects such as task requirement/instruction to the tones, orders of single- and dual-task blocks, tone location levels, tone durations, and tone frequencies. A pitch discrimination to the

\footnotetext{
${ }^{1}$ Post hoc analyses in each ANOVA of each experiment were conducted using the Bonferroni correction for multiple comparisons. Also, GreenhouseGeisser-corrected degrees of freedom were reported whenever the assumption of sphericity was violated.
}

tones was required in Experiments 1a, 1b, 3, and 4, whereas a duration reproduction to the tones was required in Experiment 5. The tone was either absent or present but ignored in Experiment 2. Experiments 1a \& 1b only differed in the orders of single- and dual-task blocks in that participants performed single-task blocks first in Experiment 1a and dual-task blocks first in Experiment $1 \mathrm{~b}$. A speeded response was required for the pitch discrimination task in Experiments $1 \mathrm{a}, 1 \mathrm{~b}$, and 3, whereas an unspeeded response given at the end of a trial for the pitch discrimination and the duration reproduction was required in Experiments 4 and 5, respectively. The tone location levels in percentage were 50/33, 70/60, and 90/87 for short/long intervals in Experiments 1a, 1b, 2, and 3, whereas they were 25/25, 50/50, and 75/75 in Experiments 4 and 5. A 50-ms tone was used in Experiment 1a, 1b, 2, and 4, whereas it was either $50 \mathrm{~ms}$ or $100 \mathrm{~ms}$ in Experiment 3 . Experiment 5 used larger tone durations, i.e., $300 \mathrm{~ms}$ or $400 \mathrm{~ms}$ for short intervals and $525 \mathrm{~ms}$ or $700 \mathrm{~ms}$ for long intervals, to fulfill the requirements of the duration reproduction task. The tone frequency was task-irrelevant in a duration reproduction task and kept as $1,000 \mathrm{hz}$ in Experiment 5, whereas it was either $1,020 \mathrm{~Hz}$ or $985 \mathrm{~Hz}$ in all the other experiments.

\section{Summarized results for all the experiments}

Table 2 summarizes the main findings of the six experiments in the present study, including the magnitudes and significances of the RT-based orienting effects and the RT-/slopebased location effects. The magnitude of the orienting effect was defined as the RT difference between the invalid and valid cue conditions. The magnitude of the location effect on $\mathrm{RT}$ was defined as the RT difference between the late and the early location levels. The magnitude of the location effect on 
Table 2 Summarized results (i.e., magnitudes and significances of orienting effects and location effects) of the dual-task-stimuli (i.e., a white circle target and an interference tone) paradigms in all experiments (see text for more details)

\begin{tabular}{|c|c|c|c|c|}
\hline \multirow[t]{2}{*}{ Experiment } & \multirow[t]{2}{*}{ Condition } & \multirow[t]{2}{*}{ Orienting effect (ms) } & \multicolumn{2}{|l|}{ Location effect } \\
\hline & & & $\mathrm{RT}(\mathrm{ms})$ & Slopes \\
\hline \multirow[t]{2}{*}{$1 \mathrm{a}$} & $\begin{array}{l}\text { Short interval } \\
\text { dual task }\end{array}$ & $8.893 *(3.748)$ & $34.265 *(6.242)$ & $0.857 *(0.156)$ \\
\hline & $\begin{array}{l}\text { Long interval } \\
\text { dual task }\end{array}$ & $1.529(2.821)$ & $22.338 *(7.043)$ & $0.414 *(0.130)$ \\
\hline \multirow[t]{2}{*}{$1 b$} & $\begin{array}{l}\text { Short interval } \\
\text { dual task }\end{array}$ & $6.254 *(2.368)$ & $35.034 *(4.444)$ & $0.876^{*}(0.111)$ \\
\hline & $\begin{array}{l}\text { Long interval } \\
\text { dual task }\end{array}$ & $0.356(2.355)$ & $11.140 *(3.483)$ & $0.206 *(0.064)$ \\
\hline \multirow[t]{2}{*}{2} & $\begin{array}{l}\text { Short Interval } \\
\text { Tone-ignored }\end{array}$ & $22.425 *(4.388)$ & $7.116(3.178)$ & $0.178 *(0.079)$ \\
\hline & $\begin{array}{l}\text { Long Interval } \\
\text { Tone-ignored }\end{array}$ & $-0.095(1.916)$ & $5.783(2.881)$ & $0.107(0.053)$ \\
\hline \multirow[t]{4}{*}{3} & $\begin{array}{l}\text { L-S order } \\
\text { short tone }\end{array}$ & $5.495 *(1.674)$ & $12.238(2.855)$ & $0.289 *(0.065)$ \\
\hline & $\begin{array}{l}\text { L-S order } \\
\text { long tone }\end{array}$ & $2.170(1.982)$ & $19.033 *(1.907)$ & $0.438 *(0.048)$ \\
\hline & $\begin{array}{l}\text { S-L order } \\
\text { short tone }\end{array}$ & $1.205(2.985)$ & $30.773 *(9.84)$ & $0.710 *(0.226)$ \\
\hline & $\begin{array}{l}\text { S-L order } \\
\text { long tone }\end{array}$ & $3.606(2.005)$ & $18.675 *(7.493)$ & $0.436 *(0.172)$ \\
\hline \multirow[t]{4}{*}{ Experiment 4} & $\begin{array}{l}\text { Short interval } \\
\text { single task }\end{array}$ & $44.64 *(5.606)$ & $21.507 *(4.419)$ & $0.430 *(0.088$ \\
\hline & Long intervalsingle task & $-6.574(3.883)$ & $0.440(2.705)$ & $0.009(0.054)$ \\
\hline & $\begin{array}{l}\text { Short interval } \\
\text { dual task }\end{array}$ & $26.470 *(5.926)$ & $60.874 *(7.536)$ & $1.217 *(0.151)$ \\
\hline & $\begin{array}{l}\text { Long interval } \\
\text { dual task }\end{array}$ & $-3.560(2.148)$ & $18.343 *(5.594)$ & $0.367 *(0.112)$ \\
\hline \multirow[t]{2}{*}{ Experiment 5} & Short interval & $25.839 *(3.355)$ & $57.382 *(4.441)$ & $1.148 *(0.089)$ \\
\hline & Long interval & $-1.318(2.104)$ & $34.640 *(4.065)$ & $0.693 *(0.081)$ \\
\hline
\end{tabular}

* Significant effects at the .05 level are indicated by asterisks. SEs are shown in parentheses

slope was defined as the slopes of the linear functions relating Tone location to RT.

\section{Experiments $1 \mathrm{a}$ and $1 \mathrm{~b}$ : The location effect for temporal orienting - A temporal-pulse accumulation and attentional gating-based account}

Experiments $1 \mathrm{a}$ and $1 \mathrm{~b}$ aimed to explore potential location effects for temporal orienting. Both single- and dual-task conditions were used. The single-task condition aimed to confirm that temporal orienting did occur with the current temporal parameters.

\section{Methods}

Experiments 1a and $1 \mathrm{~b}$ consisted of trials with a single task (a temporal-orienting task) and those with a dual task (a temporal-orienting task and a pitch discrimination task). For preview information, Experiment 1a found that RTs were faster in the dual-task condition than those in the single-task condition (see more details in the section Results: Experiment 1a). Considering the fact that all the participants completed the singe-task trials first and then the dual-task trials in Experiment $1 \mathrm{a}$, the above finding may suggest a potential practice effect in that the faster RTs in the dual-task condition were likely due to extra practice on the temporalorienting task during the earlier single-task blocks. Thus, a control experiment, i.e., Experiment 1 b, with a new sample of 20 participants was performed to explore this possibility. Experiment $1 \mathrm{~b}$ was similar to Experiment 1a except that participants completed the dual-task blocks on the first day and the single-task blocks on the next day, i.e., a reversed task order compared with Experiment 1a.

\section{Results: Experiment 1a}

A RT was treated as an outlier and discarded from the RT analyses if a response was made during the first $150 \mathrm{~ms}$ after the target onset, or if the RT was out by 2.5 standard deviation in each cell for each participant. Less than $6.94 \%$ of trials were 
labelled as outliers in the RT analysis using these criteria. The overall accuracy for the pitch-discrimination task across participants was $73.79 \%$.

\section{Location effect}

A $2 \times 3$ repeated-measures analysis of variance (ANOVA) was conducted on RTs of the target detection with Interval (short, long) and Tone location (early, middle, and late) as factors. The main effect of Interval was significant, F $(1,19)$ $=20.993, \mathrm{p}<0.001, \eta_{\mathrm{p}=0.525}^{2}$ RTs for the 3,500-ms interval were significantly faster than those for the 2,000-ms interval (mean difference $=-17.560 \mathrm{~ms}, \mathrm{SE}=3.832 \mathrm{~ms}, \mathrm{p}<.001$, Cohen's $d=-0.396)$. The main effect of Location was also significant, $F(1.139,21.634)=19.398, p<0.001, \eta_{p}^{2}=0.505$ (see Table 2). The RTs increased with the location levels (see Fig. 2 left panel). Post hoc analyses demonstrated significant RT differences between the early and the middle tone locations (mean difference $=-7.619 \mathrm{~ms}, \mathrm{SE}=2.850 \mathrm{~ms}, \mathrm{p}=0.045$, Cohen's d = -0.207 ; see Fig. 2 left panel), between the early and the late tone locations (mean difference $=-28.301 \mathrm{~ms}, \mathrm{SE}$ $=6.367 \mathrm{~ms}, \mathrm{p}=0.001$, Cohen's $\mathrm{d}=-0.600$ ) and between the middle and the late tone locations (mean difference $=-20.682$ $\mathrm{ms}, \mathrm{SE}=4.204 \mathrm{~ms}, \mathrm{t}<0.001$, Cohen's $\mathrm{d}=-0.410)$. There was a significant interaction between Tone location and Interval, $\mathrm{F}$ $(1.958,37.202)=6.030, p=0.005, \eta_{p}^{2}=0.241$. Simple effect analyses showed that when the interval was short $(2,000 \mathrm{~ms})$, there were significant RT differences between the early and the middle tone locations (mean difference $=-12.425 \mathrm{~ms}, \mathrm{SE}=$ $4.213 \mathrm{~ms}, \mathrm{p}=0.025$, Cohen's $\mathrm{d}=-0.272$ ), between the early and the late tone locations (mean difference $=-34.265 \mathrm{~ms}, \mathrm{SE}$
$=6.242 \mathrm{~ms}, \mathrm{p}<0.001$, Cohen's $\mathrm{d}=-0.640)$, and between the middle and the late tone locations (mean difference $=-21.840$ $\mathrm{ms}, \mathrm{SE}=3.436 \mathrm{~ms}, \mathrm{p}<0.001$, Cohen's $\mathrm{d}=-0.377$ ). In contrast, when the interval was long $(3,500 \mathrm{~ms})$, there were no significant RT differences between the early and the middle tone locations ( $\mathrm{p}=0.575$ ), but significant RT differences between the early and the late tone locations (mean difference $=-22.337 \mathrm{~ms}, \mathrm{SE}=7.043 \mathrm{~ms}, \mathrm{p}=0.015$, Cohen's $d=-0.533$ ) and between the middle and the late tone locations (mean difference $=-19.524 \mathrm{~ms}, \mathrm{SE}=5.488$ ms, $\mathrm{p}=0.006$, Cohen's $\mathrm{d}=-0.443)$. In addition, the slopes for the two intervals were calculated and compared in a paired-samples t-test (two-sided). The results showed that the slope in the short interval (mean $=0.857, \mathrm{SE}=$ $0.156)$ was significantly larger than the slope in the long interval $($ mean $=0.414, \mathrm{SE}=0.130), \mathrm{t}(19)=5.180, \mathrm{p}<.001$, Cohen's $\mathrm{d}=0.671$, mean difference $=.443, \mathrm{SE}=.086$. Thus, the magnitude of the location effect in terms of the slopes between the RTs and the locations was stronger in the short interval than that in the long interval.

\section{Temporal-orienting effect}

Regarding the temporal-orienting effect, a repeated-measures ANOVA with three factors, i.e., Task, Cue validity, and Interval, was conducted on RTs to the targets. The main effect of Task was significant, $F(1,19)=5.049, p=0.037, \eta_{p}^{2}=$ 0.210 . RTs were slower for the single-task condition than for the dual-task condition (mean difference $=13.706 \mathrm{~ms}, \mathrm{SE}=$ $6.100 \mathrm{~ms}, \mathrm{p}=0.037$, Cohen's $d=0.320$ ). The main effect of Cue validity reached significance, $\mathrm{F}(1,19)=19.043, \mathrm{p}<$

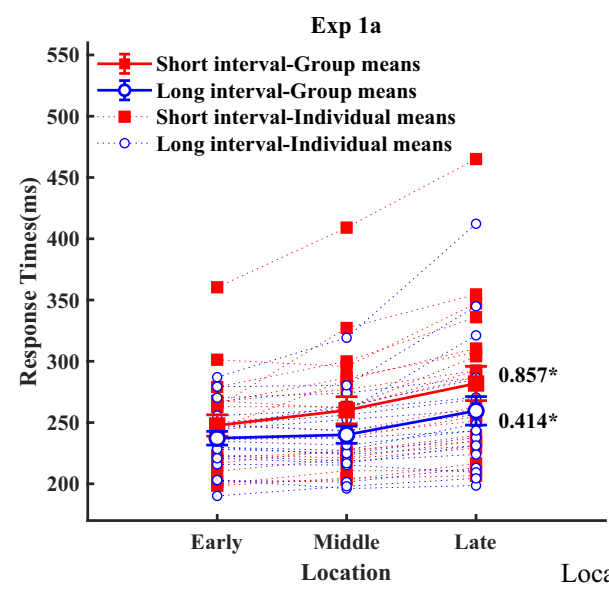

Location effect

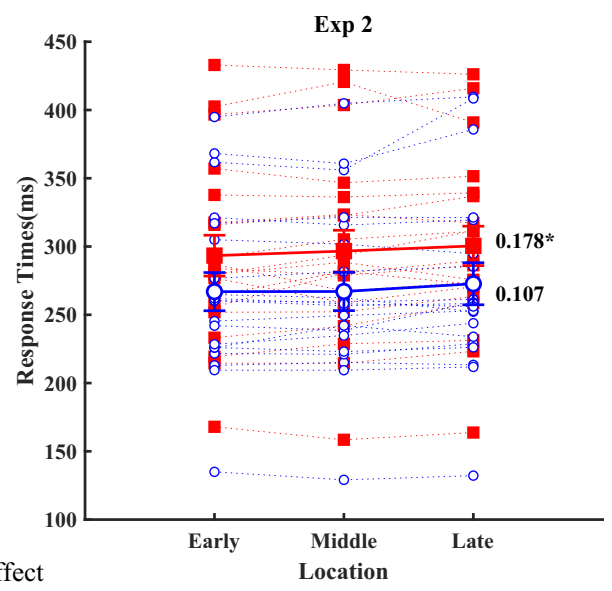

Fig. 2 The location effect for temporal orienting in the dual-task condition in Experiment 1a (left) and in the tone-ignored condition of Experiment 2 (right). Individual and group means of response times (RTs) at each location level of the short and long cue-target intervals were shown. Dashed and solid lines were used to connect individual and group means across different location levels. Error bars are SEs for group means. For the dual-task condition in Experiment 1a (left), RTs increased significantly with the location levels for both the short and the long intervals. However, for the tone-ignored condition of Experiment 2 (right), RTs slightly increased with the location levels for the short but not the long interval. The mean slope of a Tone location - RT function was labelled next to each function (calculated from the best linear fit of the three-point line). Asterisks indicate a mean slope significant at a .05 level 

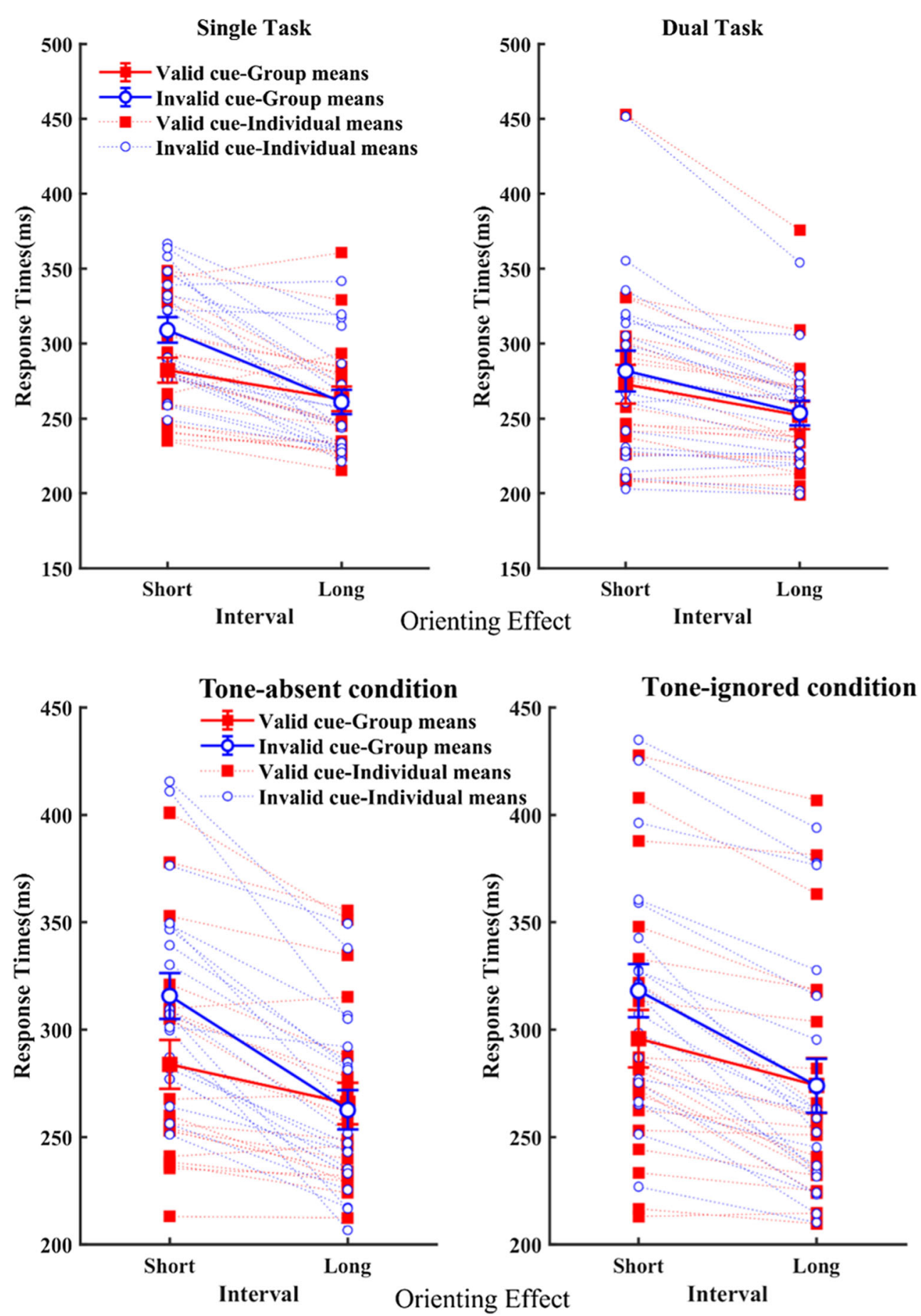

Fig. 3 The temporal orienting effect for temporal orienting in Experiment 1a (upper panel) and Experiment 2 (lower panel). Individual and group means of response times (RTs) of the target detection were shown. Dashed and solid lines were used to connect individual and group means across the short and long cue-target intervals. Error bars are SEs for group means. RTs were significantly faster for the valid than the invalid cues at the short but not the long intervals. In Experiment 1a, the magnitude of this validity effect was attenuated in the dual-task condition (upper right panel), as compared to that in the single-task condition (upper left panel). However, in Experiment 2, the magnitude of the validity effect was similar for the tone-absent (lower left panel) and the tone-ignored conditions (lower right panel)
$0.001, \eta_{p}^{2}=0.501$. RTs for the invalid cues were slower than those for the valid cues (mean difference $=8.804 \mathrm{~ms}$, $\mathrm{SE}=2.017 \mathrm{~ms}, \mathrm{p}<0.001$, Cohen's $\mathrm{d}=0.216)$. The main effect of Interval was also significant, F $(1,19)=78.079$, p < $0.001, \eta_{p}^{2}=0.804$. RTs for the short intervals were slower than those for the long intervals (mean difference $=28.883$ 
$\mathrm{ms}, \mathrm{SE}=3.269 \mathrm{~ms}, \mathrm{p}<0.001$, Cohen's $\mathrm{d}=0.702)$. The interaction between Cue validity and Interval was significant, $F(1,19)=10.325, p=0.005, \eta_{p}^{2}=0.352$ (see Fig. 3, upper panel). For the short intervals, RTs for the invalid cues were slower than those for the valid cues (mean difference $=17.869 \mathrm{~ms}, \mathrm{SE}=3.675 \mathrm{~ms}, \mathrm{p}<0.001$, Cohen's $d=0.387$ ). However, for the long intervals, RTs for the invalid cues were not significantly different from those for the valid cues $(p=.937)$. Most importantly, the three-way interaction of Cue validity $\times$ Interval $\times$ Task was significant, $F(1,19)=5.617, p=0.029, \eta_{p}^{2}=$ 0.228 (see Fig. 3, upper panel). Simple effect analyses showed that when the interval was short, participants responded faster to the cue valid than to the cue invalid trials for the single- (mean difference $=-26.845 \mathrm{~ms}, \mathrm{SE}=$ $5.520 \mathrm{~ms}, \mathrm{p}<0.001$, Cohen's $\mathrm{d}=-0.699)$ and the dual(mean difference $=-8.893 \mathrm{~ms}, \mathrm{SE}=3.748 \mathrm{~ms}, \mathrm{p}<0.05$, Cohen's $d=-0.146)$ task conditions. Moreover, these validity effects at the short intervals were more salient in the single-task condition relative to the dual-task condition. However, no such effects were observed at the long intervals in either the single- or the dual-task conditions, ps $>.05$.

\section{Results: Experiment 1b}

Due to the similar experimental design to Experiment 1a and space limitations, the results of Experiment $1 \mathrm{~b}$ are not fully reported here. In summary, consistent with Experiment 1a, a similar location effect was found, $\mathrm{F}(2,38)=37.522, \mathrm{p}<0.001$, $\eta_{\mathrm{p}}^{2}=0.664$. The fastest, intermediate, and slowest RTs on target detection were found for the early (mean $=246.501$ $\mathrm{ms}, \mathrm{SE}=4.438 \mathrm{~ms}$ ), the middle $(\mathrm{mean}=253.111 \mathrm{~ms}, \mathrm{SE}=$ $4.810 \mathrm{~ms}$ ) and the late (mean $=269.588 \mathrm{~ms}, \mathrm{SE}=6.366 \mathrm{~ms}$ ) tone locations, respectively. Consistent with Experiment 1a, there was also a significant interaction between Tone Location and Interval, $F(2,38)=18.099, p<0.001, \eta_{\mathrm{p}}^{2}=$ 0.488 . Further slope analysis confirmed that there was a significant difference in slopes between the short intervals (mean = $0.876, \mathrm{SE}=0.111)$ and the long intervals (mean $=0.206, \mathrm{SE}=$ 0.065 ), with a larger location effect for the short intervals relative to the long intervals, mean difference $=0.670, \mathrm{SE}=0.108$, $\mathrm{p}<0.001$, Cohen's $d=1.605$. Similarly, a temporal orienting effect (in terms of a significant interaction of Cue validity $x$ Interval, $\left.\mathrm{F}(1,19)=10.853, \mathrm{p}=0.004, \eta_{\mathrm{p}}^{2}=0.364\right)$ was found, similar to Experiment 1a.

Critically, participants' RTs were indeed slower in the single-task condition as compared to the dual-task condition (mean difference $=14.710 \mathrm{~ms}, \mathrm{SE}=6.833 \mathrm{~ms}, \mathrm{p}$ $<0.05$, Cohen's $d=0.592$ ), consistent with the corresponding effect in Experiment 1a (mean difference $=$ $13.706 \mathrm{~ms}, \mathrm{SE}=6.100 \mathrm{~ms}$ ).

\section{Experiment 2: A controlled attentional reallocation rather than an automatic attentional shift}

There are other alternative explanations for the location effect. For example, one hypothesis could be that the location effect was independent of controlled attentional reallocation (aiming to monitor the occurrence of the interference stimuli), and was actually related to automatic shifts of attention triggered by the non-temporal interference stimuli per se (Macar, 2002). The automatic attentional shift may reset the process of temporalpulse accumulation or make part of the already accumulated pulses lost. This provided an important rationale for the design of Experiment 2 to verify to what extent the location effect revealed in Experiments 1a and $1 \mathrm{~b}$ could be explained by an automatic attentional shift triggered by task-irrelevant interference stimuli.

\section{Methods}

\section{Stimuli and procedure}

Stimuli and procedure in Experiment 2 were similar to Experiments 1a and $1 \mathrm{~b}$ except that participants were instructed to ignore the tones whenever they occurred (see Table 1). Thus, the stimuli (and the instructions) of the tone-absent condition in Experiment 2 were exactly the same as the singletask conditions in Experiments 1a and 1b, whereas the stimuli (but not the instructions) of the tone-ignored condition in Experiment 2 was equal to that of the dual-task condition in Experiments $1 \mathrm{a}$ and $1 \mathrm{~b}$. The order of the tone-absent/-ignored blocks was counterbalanced across participants.

\section{Results}

The screening procedure for RT outliers was the same as that in Experiments 1a and 1b. Less than $6.27 \%$ of trials were labelled as outliers in the RT analysis.

\section{Location effect and temporal-orienting effect}

A repeated-measures ANOVA on RTs to the targets for the tone-ignored condition was conducted. The main effects of Interval, $F(1,19)=71.030, p<0.001, \eta_{p}^{2}=0.789$, and Location, $F(2,38)=11.066, p<0.001, \eta_{p}^{2}=0.368$ (see Table 2), were both significant. There was no two-way interaction, $p>.05$. Slopes at the short $($ mean $=0.178, \mathrm{SE}=0.079$, $\mathrm{p}<.039$ ) and the long (mean $=0.107, \mathrm{SE}=0.053, \mathrm{p}>.05$ ) intervals were calculated and a paired samples t-test (twosided) showed no significant difference, $\mathrm{p}=0.542$.

A temporal orienting effect (in terms of a significant interaction of Cue validity $\times$ Interval, $F(1,19)=39.797, p<0.001$, $\eta_{p}^{2}=0.677$ ) was found, similar to Experiment 1a. However, 
different from Experiment 1a, the three-way interaction of Cue validity $\times$ Interval $\times$ Task was not significant, $\mathrm{p}>.05$ (see Fig. 3 lower panel).

\section{Comparisons of the location effects between Experiments 1a and 2}

An ANOVA with one between-subject factor, i.e., Experiment (Experiment 1a-Tone-Processed dual-task condition vs. Experiment 2-Tone-ignored condition, see Fig. 2), and two within-subject factors, i.e., Interval (short, long) and Tone location (early, middle, and late), was conducted on RTs of the target detection. Significant main effects were found for Interval, $F(1,38)=80.585, p<0.001, \eta^{2}=0.680$, with faster RTs for the long intervals compared to the short intervals, and for Location, $F(2,76)=26.810, p<0.001, \eta^{2} p=0.414$. Most critically, the interaction between Tone location and Experiment was significant, $\mathrm{F}(2,76)=10.593$, $\mathrm{p}<0.001$, $\eta^{2} p=0.218$. Analysis of simple effects showed that when participants were instructed to process a tone and complete the pitch discrimination task (Experiment 1a), they were quicker to detect a subsequent target with the tone occurring at a relatively-early than a relatively-late time point. This location effects emerged in the paired comparisons between the early and the middle locations $(p=.004$, Cohen's $d=-0.267)$, between the early and the late locations ( $p=.000$, Cohen's $d=$ -0.822 ) and between the middle and the late locations ( $p=$ .000 , Cohen's $\mathrm{d}=-0.552$ ). However, when participants were instructed to ignore the tones (Experiment 2), the RTs to detect a subsequent target were not significantly different for any paired comparison between a relatively early tone and a relatively late tone ( $\mathrm{ps}>.05$ ). This interaction suggested that the location effect was much stronger for the tone-processed condition in Experiment 1a than for the tone-ignored condition in Experiment 2.

\section{Discussion of Experiments 1 and 2}

The results of Experiments 1a and 1b demonstrated clear location effects for temporal orienting in the dual-task conditions. A linear relationship between RT of temporal orienting and location levels was observed, suggesting that the internal representation of the temporal orienting likely possessed a feature of online temporal-pulse accumulation and attentional gating with the elapse of time. The framework of the attentional-gate model (Zakay \& Block, 1997) seems to be a most appropriate and parsimonious choice to account for this feature. Similar logic and theoretic framework were also adopted to explain the location effect for explicit timing (Casini \& Macar, 1997; Fortin \& Tremblay, 2006). Here, we proposed a temporal-pulse accumulation and attentional gating-based mechanism, similar to that for explicit timing, to account for the location effect for temporal orienting. Namely, the performance of the target detection was directly related to how well the temporal orienting to the target was constructed. When the to-be-discriminated tone was closer to the target, attention was deviated from the temporal-pulse accumulation for a longer time to monitor the occurrence of the interference tones, leading to a greater loss of the temporal pulses and a slower motor response to the subsequent target. The magnitude of the disruption depended on how late the tone would appear - hence leading to a location effect for temporal orienting, similar to that for explicit timing (Casini \& Macar, 1997). At the same time, strong temporal orienting effects were revealed in Experiments $1 \mathrm{a}$ and $1 \mathrm{~b}$ for both the single- and the dual- task conditions, confirming the validity of the intervals $(2,000 \mathrm{~ms}$ and $3,500 \mathrm{~ms})$ used in the present study in terms of eliciting temporal orienting.

Both Experiment 1a and Experiment $1 \mathrm{~b}$ demonstrated that RTs were indeed slower in the single-task condition as compared to the dual-task condition. Thus, we can draw a conclusion that the quicker RTs for the dual-task condition relative to the single-task condition is a genuine effect, rather than an artefact from excessive practice. This effect might be related to the role of action execution in the dual-task condition. The response of the pitch discrimination involved a component of action execution in Experiments $1 \mathrm{a}$ and $1 \mathrm{~b}$. Some neutral substrates, such as basal ganglia (Artieda, Pastor, Lacruz, \& Obeso, 1992; Bueti, Walsh, Frith, \& Rees, 2008; Coull \& Nobre, 1998; Meck, 2005) and supplementary motor cortex (Coull, Cheng, \& Meck, 2011; Grondin, 2010; Schwartze, Rothermich, \& Kotz, 2012), were found to be jointly activated by an action execution and a temporal processing. Thus, the action execution component in the dual task might increase the activation of these brain areas in the first place which in turn boosted the precision of a subsequent temporal processing (Fautrelle, Mareschal, French, Addyman, \& Thomas, 2015). The influence of the action execution component on an explicittiming task was also observed in an earlier study (Haggard, Clark, \& Kalogeras, 2002).

The combined analysis on Experiments 1a and 2 ruled out the possibility that the location effect was dominantly due to automatic shifts of attention. The location effect was significant in the target-processed condition in Experiment 1a, but not in the target-ignored condition in Experiment 2, suggesting that the location effect was primarily due to a controlled attentional shift in order to monitor the occurrence of an interference stimulus, rather than an automatic shift of attention by a task-irrelevant stimulation per se. The above results echoed with a previous study in explicit timing (Champagne \& Fortin, 2008), which suggested that an automatic shift of attention was not the prime factor for the location effect for explicit timing. 


\section{Experiment 3: The location effect is sensitive to processing demand/task difficulty}

Using short and long tones in a pitch-discrimination task, Champagne and Fortin (2008) found that a larger location effect emerged in conditions when participants were tested first with short tones, rendering the pitch discrimination more processing demanding, relative to conditions when participants were tested first with long tones. Experiment 3 aimed to explore whether similar effects also occur for temporal orienting, i.e., whether and how processing demand modulates the location effect for temporal orienting. The paradigm was adapted from the dual-task condition of Experiments $1 \mathrm{a}$ and $1 \mathrm{~b}$ by varying the difficulties of the pitch discrimination. On the basis that temporal orienting involves controlled processing that is based on temporalpulse accumulation, we expected that more attentional resources would be deviated from the temporal orienting for a more difficult interference task, leading to a larger location effect.

\section{Methods}

\section{Experimental task and stimuli}

In order to manipulate the processing demands of the nontemporal pitch discrimination, two lengths of tones were used in Experiment 3 (see Table 1), $50 \mathrm{~ms}$ for the short-tone condition with a relatively higher task difficulty and $100 \mathrm{~ms}$ for the long-tone condition with a relatively lower task difficulty. The former condition should have a higher processing demand than the latter one. The testing order of the short/ difficult $(50 \mathrm{~dB}, 985 / 1,020 \mathrm{~Hz}, 50 \mathrm{~ms})$ and the long/easy (50 $\mathrm{dB}, 985 / 1,020 \mathrm{~Hz}, 100 \mathrm{~ms}$ ) tone blocks was counterbalanced across participants. There were no single-task blocks in this experiment. Other aspects were the same as Experiments 1a and $1 b$.

\section{Procedure}

There were 12 experimental blocks, six for the "difficult" and six for the "easy" conditions. Each block consisted of 48 trials, including 36 cue-valid and 12 cue-invalid ones, i.e., a $75 \%$ cue validity. Half the participants were tested with the short-tone blocks first, then the long-tone blocks in a second session (denoted as SL order), whereas the other half participants were tested in the reverse order (denoted as LS order). The two sessions were separated by 1 day. Each experimental session took about $30 \mathrm{~min}$. Other aspects were the same as Experiments 1a and $1 \mathrm{~b}$.

\section{Design and data analyses}

Experiment 3 constituted a mixed experimental design. The between-subjects variable was Tone order with two levels (SL and LS). The within-subjects variables were Tone location (early, middle, late), Tone duration (long, short), Cue validity (valid, invalid), and Interval (short, long). Similar to previous experiments, separate ANOVAs with corresponding variables were conducted to analyze the location effect and the temporal orienting effect. Slopes were also analyzed for the location effect.

\section{Results}

The screening procedure for RT outliers was the same as that in Experiments 1a and 1b. Less than $7.83 \%$ of trials were labelled as outliers in the RT analysis. A pairedsamples t-test (two-sided) showed that the error rate was significantly higher for the short- $(20.69 \%)$ than the longtone $(12.63 \%)$ conditions, $t(22)=3.729, p=0.001$, Cohen's $d=0.509$, indicating an effective manipulation of the task difficulty.

\section{Location effect, order effect, and task difficulty-sensitivity}

For the location effect, a four-way mixed-design ANOVA with Tone location, Interval, Tone duration, and Tone order as factors was conducted on RTs of the target detection. The main effect of Interval was significant, F $(1,21)=27.556$, $\mathrm{p}<$ $0.001, \eta_{p}^{2}=0.568$, with faster RTs for the long intervals than the short intervals. The main effect of Tone duration was significant, $F(1,21)=4.701, p<0.05, \eta_{p}^{2}=0.183$, with faster RTs for the long tones than the short tones. The main effect of Tone location was also significant, $F(1.07,22.479)=24.453$, $\mathrm{p}<0.001, \eta_{\mathrm{p}}^{2}=0.538$, demonstrating a location effect (see Table 2). More importantly, the two-way interaction between Interval and Tone location was significant, $\mathrm{F}(1.369,28.751)$ $=11.002, p=0.001<0.05, \eta_{p}^{2}=0.344$. For a short interval $(2,000 \mathrm{~ms}), \mathrm{RTs}$ differed between any two different tone locations (ps < .05). However, for a long interval $(3,500 \mathrm{~ms})$, RTs only differed between the early and the late locations and between the middle and the late locations ( $\mathrm{ps}<.05$ ), but not between the early and the middle locations $(\mathrm{p}>.05)$.

There was also a significant interaction between Tone duration and Tone order, $F(1,21)=20.023, p<0.001, \eta_{p}^{2}=$ 0.488 . RTs were significantly faster in the LS order than in the $\mathrm{SL}$ order for the short tones (mean difference $=-27.521 \mathrm{~ms}$, $\mathrm{SE}=11.981 \mathrm{~ms}, \mathrm{p}<.05$, Cohen's $\mathrm{d}=-0.924)$, but not for the long tones, $p>.05$. In other words, an order effect (better performance for a condition when it was tested in the second experimental session than when the same condition was tested in the first experimental session) was observed for the short but not the long tones, leading to an asymmetrical order 
effect between the short and the long tones. This two-way interaction also suggested that the RT differences between the short and the long tones were significant only for the SL order condition (mean difference $=25.295 \mathrm{~ms}$, SE = $5.501 \mathrm{~ms}, \mathrm{p}<.001$, Cohen's $\mathrm{d}=0.978)$, but not for the LS order condition $(\mathrm{p}>.05)$.

A three-way interaction between Tone location, Tone duration, and Tone order was found, $F(2,42)=10.021, p=$ $0.001<0.05, \eta_{\mathrm{p}}^{2}=0.323$ (see Fig. 4). For the short-tone condition, trend analysis demonstrated significant linear trends in both the LS order condition, $\mathrm{F}(1,11)=99.609, \mathrm{p}<0.001, \eta_{\mathrm{p}}^{2}$ $=0.901$ and in the SL order condition, $\mathrm{F}(1,11)=18.378, \mathrm{p}=$ $0.001<0.05, \eta_{p}^{2}=0.626$. For the long-tone condition, significant linear trends were found in the LS order condition, F ( 1 , 10) $=6.211, p=0.032, \eta_{p}^{2}=0.383$ and in the SL order condition, $\mathrm{F}(1,10)=9.780, \mathrm{p}=0.011, \eta_{\mathrm{p}}^{2}=0.494$. Moreover, the magnitude of the location effect in the form of a linear increase of RTs with tone locations was different across the different combinations of Tone duration and Tone order. The location effect was strongest with a short tone in the SL order condition. This was confirmed by further slope analysis. The ANOVA on slope data showed a significant interaction between Tone duration and Tone order, F $(1,21)$ $=12.605, p=0.002, \eta_{p=0}^{2}$ 0.375. Simple effect analyses found that the slope differences between the long and short tone durations were not statistically significant for the LS order condition, $\mathrm{p}>.05$. However, the slopes were significantly larger (mean difference $=0.274, \mathrm{SE}=0.086, \mathrm{p}=.004$, Cohen's $d=0.420$ ) in the short tones (mean slope $=0.710$,
$\mathrm{SE}=0.164)$ than in the long tones (mean slope $=0.436, \mathrm{SE}=$ 0.124 ) for the SL order condition (see Fig. 4). Thus, a significant task difficulty-sensitivity, defined as significant slope differences between the short and the long tones, was observed for the SL-order condition, but not for the LS-order condition, which was likely due to the influence from the asymmetrical order effect between the short and the long tones.

Temporal orienting effect $\mathrm{A}$ temporal orienting effect (in terms of a significant interaction of Cue validity $\times$ Interval, $\left.\mathrm{F}(1,21)=5.459, \mathrm{p}=0.029<0.05, \eta_{\mathrm{p}}^{2}=0.206\right)$ was found, similar to Experiment $1 \mathrm{a}$.

\section{Discussion}

The results of Experiment 3 confirmed the location effect revealed by Experiments 1a and $1 \mathrm{~b}$. Moreover, a significant task difficulty-sensitivity was observed for the SL-order condition when participants were tested with the short tones in the first experimental session, but not for the LS-order condition when participants were tested with the long tones in the first experimental session. The above results were likely due to the influence of the asymmetrical order effect between the short and the long tones on the task difficulty-sensitivity. More specifically, the order effect was present in the short tones but absent in the long tones. Such an order effect may reflect a general increase in training and familiarity with the task, which was more effective for conditions with higher levels

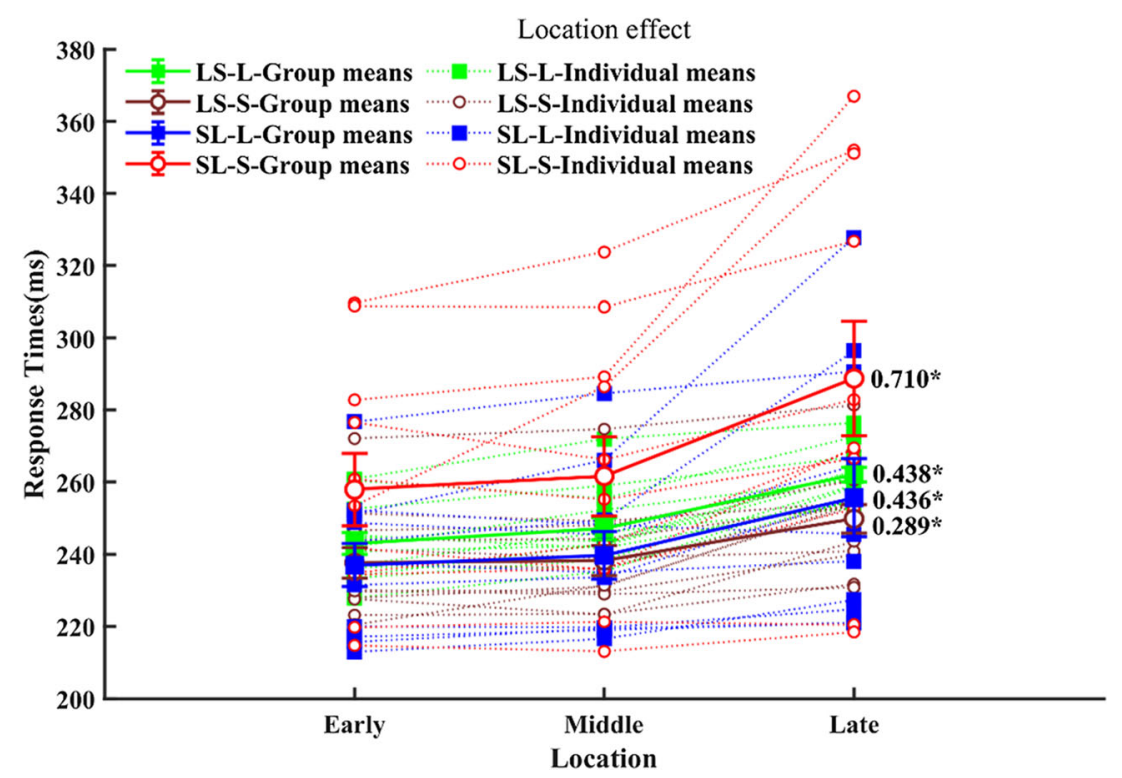

Fig. 4 The location effect for temporal orienting and its task difficultysensitivity modulated by tone order in Experiment 3 . Individual and group means of response times (RTs) at each location level of the short and long tone durations were shown with different tone orders. RTs for the target detection increased significantly with the increase of the location levels. The SL represented a tone order with the short tone block first and the LS the long tone block first. The '-L' represented a long tone duration and '-S' a short tone duration. Other aspects of the figure format are similar to Fig. 2 
of task difficulty, i.e., the pitch discrimination for the short tones. A similar asymmetrical order effect between the short and the long tones had been observed in a previous study (Champagne \& Fortin, 2008) demonstrating a task difficulty-sensitivity in the case of explicit timing. The influence of the asymmetrical order effect has been observed on both indices of slopes and RTs in the present study.

According to the framework of attention-sharing mechanisms (Fortin, 2003; Fortin \& Massé, 2000; Macar et al., 1994), attentional resource was divided between a process of the online accumulating to the temporal pulses and the other process of the online monitoring to the occurrence of a stimulus in a non-temporal-interference task (Fortin, 2003). When the processing demands of the non-temporal-interference task increased, for example by asking participants to discriminate the frequencies of tones with short durations, more attentional resource would be forced into the online monitoring process, and thus increase the magnitude of the location effect eventually.

\section{Experiment 4: Location effects with matched location levels, equal spacing and unspeeded tone responses}

Experiments 1a, 1b, and 3 demonstrated clear location effects for implicit timing. However, two further aspects in the dual-task paradigm require further attention. First, the location levels (and the spacing between neighboring location levels) were not matched between the short and long intervals in the above 3 experiments, i.e., 50\%, 70\%, and $90 \%$ (and $20 \%$ as the spacing) for the 2,000-ms interval and $33 \%, 60 \%$, and $87 \%$ (and $27 \%$ as the spacing) for the 3,500$\mathrm{ms}$ interval. It is unclear whether the location effects in Experiments 1a, 1b, and 3 are due to the unmatched location levels with unequal spacing. Thus, the first purpose of Experiment 4 was to explore whether the location effects in Experiments $1 \mathrm{a}, 1 \mathrm{~b}$, and 3 were robust when matched location levels with equal spacing were adopted for the short and long cue-target intervals.

Second, the dual-task paradigm in Experiments 1a, 1b, and 3 required participants to give two speeded responses sequentially, i.e., first to the pitch discrimination and then to the temporal orienting. According to the well-established phenomenon of the psychological refractory period (PRP; cf. Pashler, 1984, 1994), RTs to a second task could increase rapidly with decreased temporal distances between two tasks requiring speeded responses (Fan et al., 2012). Thus, it is yet to be clarified whether the location effects in Experiments 1a, $1 \mathrm{~b}$, and 3 , i.e., increased RTs of the temporal orienting with decreased temporal distances between the tones and the targets, were due to the PRP effect.

\section{Methods}

\section{Experimental task, stimuli, and procedure}

Experiment 4 had similar design as Experiments $1 \mathrm{a}$ and $1 \mathrm{~b}$ except for two aspects (see Table 1). First, the tones always appeared in three fixed time point, i.e., $500 \mathrm{~ms}, 1,000 \mathrm{~ms}$, and $1,500 \mathrm{~ms}$ for the $2,000-\mathrm{ms}$ intervals, and $875 \mathrm{~ms}, 1,750 \mathrm{~ms}$, and 2,625 $\mathrm{ms}$ for the 3,500-ms intervals. These location levels corresponded to three percentage values with equalized spacing, i.e., $25 \%, 50 \%$, and $75 \%$ (25\% as the spacing) for both intervals. Second, in order to explore the PRP-based alternative explanation to the location effect, the order of the two responses were reversed in this experiment, compared with that in Experiments 1a, 1b, and 3. More specifically, the response to the target detection now became the first one. The response of the pitch discrimination was moved into the end of a trial as the second and a unspeeded response, after the full completion of the first one. The order of the single- and dualtask blocks was counterbalanced across participants. Other aspects were the same as Experiments 1a and 1b. It is worth noting that the manipulation of the response orders here may also contribute to a further exploration on whether the reduced RTs of the target detection in the dual task relative to that in the single task (observed in Experiments 1a and 1b) was due to the response execution component of the pitch discrimination (see Discussion for more details).

\section{Results}

The screening procedure for RT outliers was the same as that in Experiments 1a and 1b. Less than $6.39 \%$ of trials were labelled as outliers in the RT analysis. The overall accuracy, i.e., proportion of correct responses, for the pitch discrimination task across participants was $87.67 \%$.

\section{Location effect}

A $2 \times 2 \times 2 \times 3$ repeated-measures ANOVA was conducted on RTs of the target detection with Task (single task, dual task), Interval (short, long), Cue validity (valid, invalid), and Tone location (early, middle and late) as factors. Unlike Experiments $1 \mathrm{a}$ and $1 \mathrm{~b}$, Task had no main effect, $\mathrm{F}(1,27)=$ $1.370, \mathrm{p}=.252, \eta_{\mathrm{p}}^{2}=0.048$. Participants did not show a speeded response to the target detection in the dual task relative to that in the single task when the response orders in the dual task were reversed and there was no response execution before the response to the target detection.

There was a significant three-way interaction between Task, Interval, and Tone location, F $(1.501,40.537)=7.090$, $p=.05, \eta_{p}^{2}=0.208$ (see Fig. 5). Simple effect analyses demonstrated a significant effect of the tone location for both the short $\left(F(2,54)=63.550, p<.001, \eta_{p}^{2}=0.702\right)$ and the long 

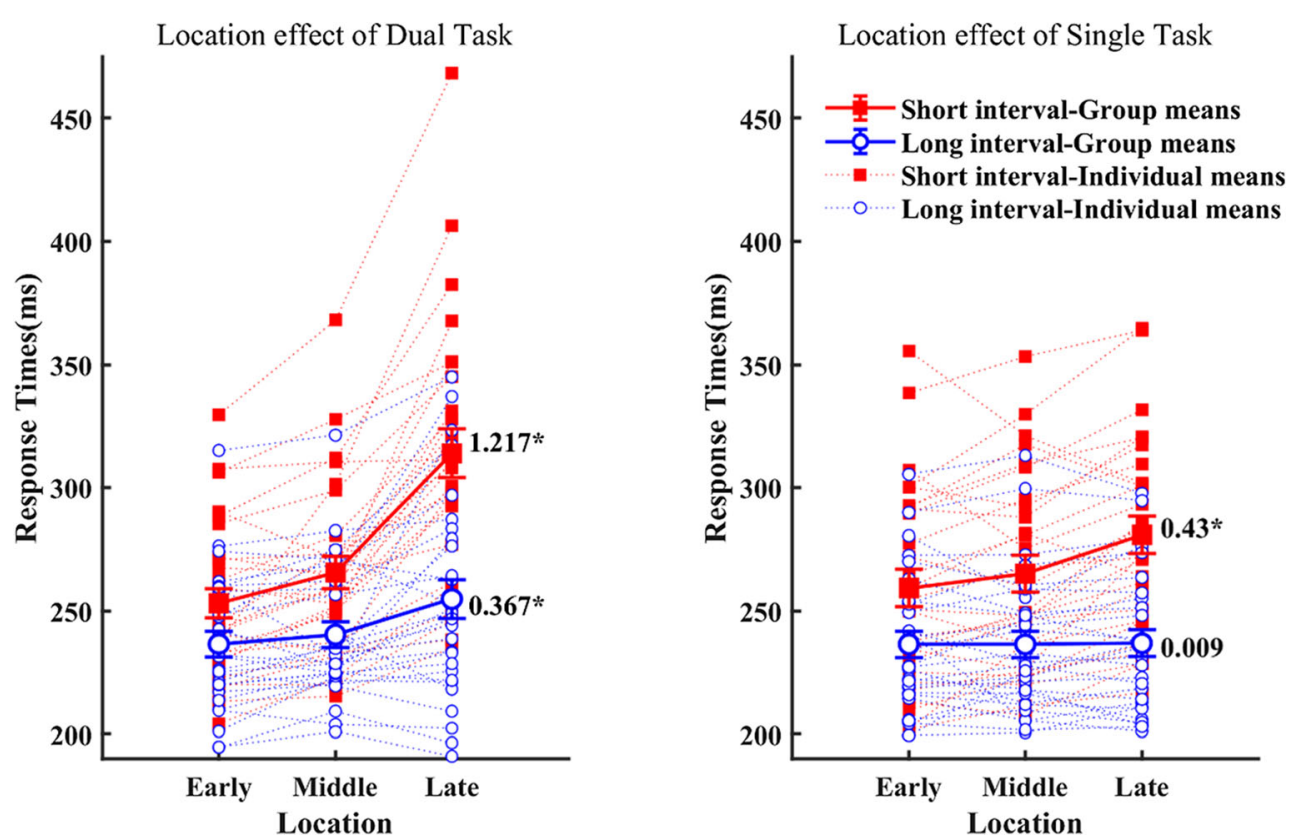

Fig. 5 The location effect for temporal orienting in Experiment 4. Individual and group means of response times at each location level of the short- and long-interval conditions are shown. Other aspects of the figure format are similar to Fig. 2

$\left(\mathrm{F}(2,54)=8.962, \mathrm{p}<.001, \eta_{\mathrm{p}}^{2}=0.249\right)$ intervals in the dualtask condition. Conversely, significant effect of the tone location was only found for the short $(\mathrm{F}(2,54)=19.079, \mathrm{p}<.001$, $\left.\eta_{p}^{2}=0.414\right)$ but not the long $(p=.973)$ intervals in the singletask condition.

In addition, a repeated-measures ANOVA with Task and Interval as factors was performed on the measure of slopes. The interaction between Interval and Task reached significance, $F(1,27)=8.804, p=.006, \eta_{p}^{2}=0.246$. Simple effect analyses showed that slopes were larger in the dual task than in the single task for both the short intervals (mean difference $=.787, \mathrm{SE}=.156$, Cohen's $\mathrm{d}=1.182$ ) and the long intervals (mean difference $=.358, \mathrm{SE}=.113$, Cohen's $\mathrm{d}=0.756$ ). The slope differences were larger for the short interval than for the long interval. The magnitude of the location effect was larger in the short interval than that in the long interval.

\section{Temporal orienting effect}

Similarly, a temporal orienting effect (in terms of a significant interaction of Cue validity $\times$ Interval, $F(1,27)=57.843, p<$ $.001, \eta_{\mathrm{p}}^{2}=0.682$ ) was found, similar to Experiment $1 \mathrm{a}$. There was also a significant three-way interaction between Task, Interval, and Cue validity, $F(1,27)=10.927, p=.003, \eta_{p}^{2}$ $=0.288$.

\section{The influence of temporal orienting on pitch discrimination}

A $2 \times 2 \times 2 \times 3$ repeated-measures ANOVA was conducted on correct rates of the pitch discrimination with Task (single, dual), Cue-target Interval (short, long), Cue validity (Valid, Invalid), and Tone location (early, middle and late) as factors. There were no significant main effects or interactions (ps > .05). Particularly, there was no significant locational modulation on the pitch discrimination. Thus, the location effect in Experiment 4 was one way, i.e., there was only locational modulation from the pitch discrimination to the temporal orienting but not the other way around.

\section{Discussion}

The results showed a significant location effect for temporal orienting, indicating that the location effects in Experiments $1 \mathrm{a}, 1 \mathrm{~b}$, and 3 were robust and can be replicated when matched location levels with equal spacing for both the short and long cue-target intervals were adopted. A critical difference between Experiment 4 and Experiments $1 \mathrm{a}$ and $1 \mathrm{~b}$ is that an unspeeded response given at the end of a trial was required for the pitch discrimination in Experiment 4, whereas a speeded response was required for the pitch discrimination in Experiments $1 \mathrm{a}$ and $1 \mathrm{~b}$. Thus, before the speeded response to the target detection, a response execution component of the tone pitch discrimination was absent in Experiment 4 but present in Experiments 1a and 1b. If our explanation to the origin of RT differences between the single and dual tasks in Experiments $1 \mathrm{a}$ and $1 \mathrm{~b}$ is true, the facilitating influence of the action execution on the performance of temporal orienting should be observed in Experiments $1 \mathrm{a}$ and $1 \mathrm{~b}$ but not in Experiment 4. These predictions were confirmed here. However, we acknowledge that the present study can only 
provide evidence to our explanation from a behavioral perspective. It might be worth future studies using neuroimaging approaches, such as fMRI (functional magnetic resonance imaging) technology, to explore this issue in a more direct way.

Based on the stimulus arrangements in Experiments 1a, 1b, and 3 and the results of Experiment 4, it is unlikely that the location effect found in Experiments $1 \mathrm{a}$ and $1 \mathrm{~b}$ can be accounted for by the well-established PRP effect (Pashler, 1984, 1994). First, according to our design and data analysis approach in Experiments 1a, 1b, and 3, the completion of a valid response to the pitch discrimination occurred before the target presentation. Thus, it was lack of a temporal overlap between the pitch discrimination and the target detection. This suggests that the location effect found here was not likely due to an information-processing "bottleneck" in which the processing of the pitch discrimination temporarily prevents the execution of crucial steps of the other temporally overlapping task, i.e., the target detection. This information-processing "bottleneck" lies at the heart of all models for the explanation of the PRP (Meyer \& Kieras, 1997; Pashler, 1984, 1994). Second, the hallmark effect of the PRP was that the RTs to the second of two independent tasks (both require speeded responses) increased rapidly with the decrease of SOA (stimulus-onset asynchrony) between them, whereas the RTs to the first task were largely uninfluenced by the SOAs (Pashler, 1984, 1994). The manipulation of response orders in Experiment 4 can differentiate the hallmark effect of PRP and the location effect found here. In line with Experiments 1a and $1 \mathrm{~b}$, a similar location effect was found in Experiment 4 on both RT- and slope-based analyses, providing strong evidence that the location effect was not due to the PRP effect.

\section{Experiment 5: Mutual interference on temporal-pulse accumulation between implicit and explicit timing}

The purpose of this experiment was to explore the hypothesis of shared temporal-pulse accumulation between implicit and explicit timing in a more direct way by pitting the two processes against each other within a trial. Here, we used a dualtask paradigm consisting of an implicit temporal-orienting task and an explicit duration reproduction task. If each task (regardless of whether it is implicit or explicit timing) elicits an internal temporal representation that is based on temporalpulse accumulation, then the temporal-pulse-accumulation conflicts between the two tasks would occur. This could lead to the occurrence of the location effect for the interference task along with the location effect for temporal orienting, i.e., twoway location effects, contrast to the one-way location effect in Experiment 4 with single temporal-pulse accumulation involved.

\section{Methods}

\section{Experimental task and stimuli}

The paradigm in Experiment 5 involved an implicit- and an explicit-timing task (see Table 1). The implicit temporalorienting task was the same as that in Experiment 4. The explicit duration reproduction task required participants to reproduce the perceived duration of a tone $(50 \mathrm{~dB}, 1,000 \mathrm{~Hz})$ occurring between the cue and the target circle. The physical duration of the tone was $300 \mathrm{~ms}$ (short) or $400 \mathrm{~ms}$ (long) for the short cue-target interval $(2,000 \mathrm{~ms})$, and $525 \mathrm{~ms}$ (short) or $700 \mathrm{~ms}$ (long) for the long cue-target interval $(3,500 \mathrm{~ms})$. Note the ratios $(0.75)$ between the short- and long-tone durations, i.e., $300 / 400$ and $525 / 700$, were the same for the two cuetarget intervals. The temporal distances between the tone offset and the start of the reproduction phase (signaled by the onset of two red question marks on the display, see Fig. 6) were kept constant across all the trials to control the potential confounding effect of working memory. To achieve this, the temporal distances between the offset of the target circle and the start of the reproduction phase varied from a minimum of $1,100 \mathrm{~ms}$ (corresponding to the condition with the short-tone duration, the early-tone location, and the long cue-target interval) to a maximum of $3,100 \mathrm{~ms}$ (corresponding to the condition with the long tone duration, the late tone location and the short cue-target interval).

Participants were required to give their temporal orienting responses before the start of the reproduction phase (a failure to do so will nullify a trial). The minimum $1,100 \mathrm{~ms}$ interval between the disappearance of the target circle and the start of the reproduction phase was much larger than participants' average RTs in the temporal-orienting task (below $300 \mathrm{~ms}$, see Fig. 2, left panel), which guaranteed an early completion of the temporal orienting response and can effectively eliminate its interference to the subsequent duration reproduction. Participants were instructed to use their right index finger to press the spacebar to reproduce the perceived duration of the tone as long as they saw the two red question marks. The keypressing was accompanied by a pure tone $(50 \mathrm{~dB}, 1,000 \mathrm{~Hz})$, which stopped immediately after the release of the response key. The duration of reproduction was not a speeded response, thus only accuracy, i.e., as close as possible to the physical duration of the tone, was emphasized. Other aspects were the same as Experiment 4.

\section{Procedure and design}

Before the formal test, participants had to complete practice sessions for the duration of the reproduction task. At the end of each practice trial, a visual feedback informed participant whether their reproduction was up to standard or not (too short or too long). The range of up-to-standard time reproduction 


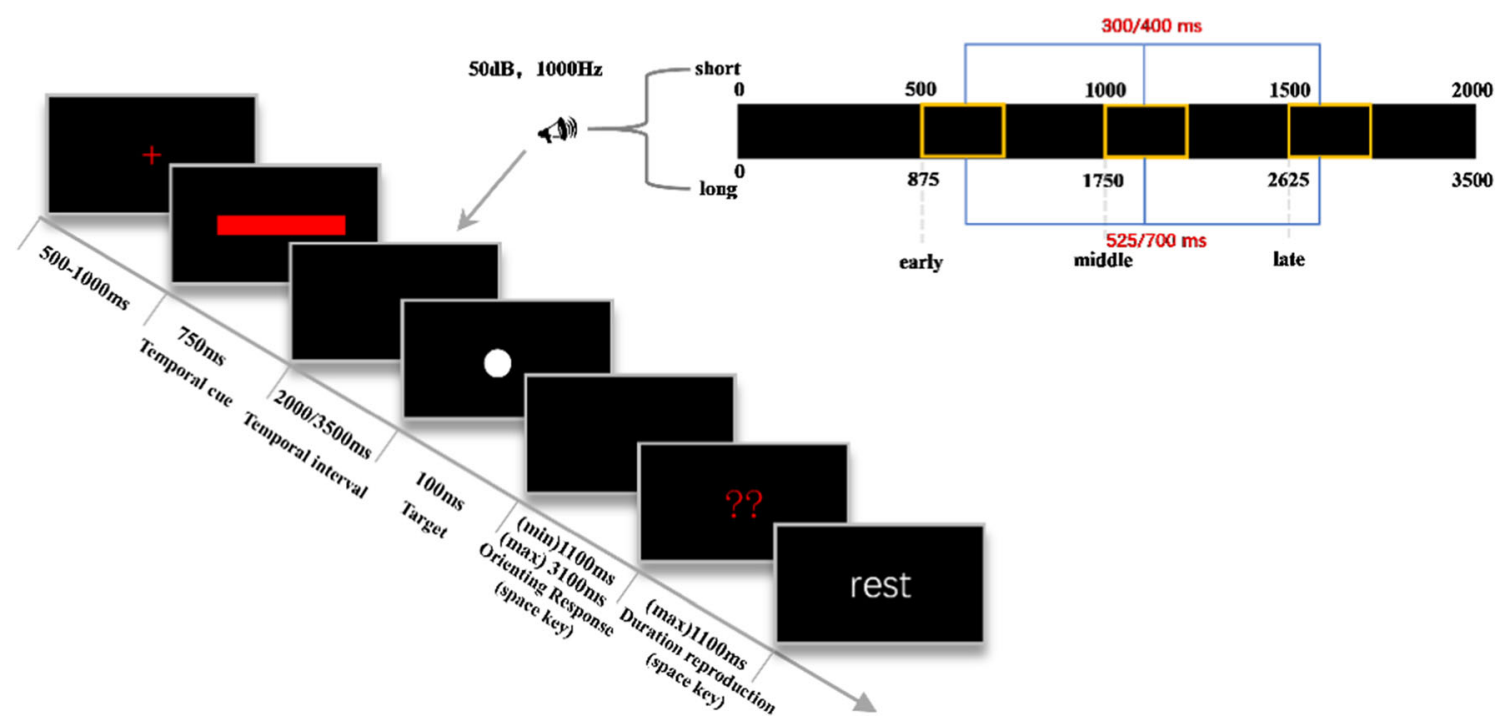

Fig. 6 Schematic representation of events for a trial in Experiment 5. The location levels (early, middle, and late) and the short/long tone durations were shown in yellow rectangle frames for the 2,000-ms and 3,500-ms interval conditions, respectively

was initially set to less than $20 \%$ deviation of the physical tone duration and dynamically adjusted by decreasing $(1.5 \%)$ or increasing $(1.5 \%)$ the range after each correct or incorrect trial. The tone durations and the location levels were fully crossed for both the cue-valid/-invalid trials. Different conditions were randomly mixed in the test. The total experiment took about $50 \mathrm{~min}$.

\section{Data analysis}

Experiment 5 manipulated four within-subject variables, i.e., Tone duration (short, long), Cue-target Interval (short, long), Cue validity (valid, invalid), and Tone location (early, middle, and late). RTs to the target detection was used to evaluate the influence of the duration reproduction on the temporal orienting. Conversely, the influence of the temporal orienting on the reproduction of the tone's duration was evaluated by the estimated-to-physical-duration ratio (RATIO). The RATIO was calculated by dividing each trial's Reproduced duration $\left(\mathrm{R}_{\mathrm{d}}\right)$ by the Stimulus/tone duration $\left(\mathrm{S}_{\mathrm{d}}\right)$ for that trial [RATIO $=\mathrm{R}_{\mathrm{d}} / \mathrm{S}_{\mathrm{d}}$ ]. This provided an index for the quantity and the direction of errors during explicit time reproduction, with coefficients above and below 1.0 being indicative of overreproduction and under-reproduction, respectively.

\section{Results}

All trials containing any missed response from the two tasks were excluded. A further two-step screening procedure was performed to remove outliers. First, a trial was treated as an outlier and discarded if its response to the temporal-orienting task was made during the first $150 \mathrm{~ms}$ after the target onset or if its duration reproduction was shorter than $100 \mathrm{~ms}$ or longer than $933 \mathrm{~ms}$. Second, a trial in any condition for any participant was labelled as an outlier if its RT to the temporal-orienting task was out of 3 standard deviations or if its reproduced duration was out of 2 standard deviations in that condition for that participant. In total, less than $6.14 \%$ trials were removed.

\section{The influence of explicit time reproduction on temporal orienting}

A $2 \times 2 \times 2 \times 3$ repeated-measures ANOVA was conducted on RTs of the target detection with Tone duration (short, long), Cue-target Interval (short, long), Cue validity (Valid, Invalid), and Tone location (early, middle and late) as factors. There was a main effect of Tone duration, $\mathrm{F}(1,27)=$ $34.595, \mathrm{p}<.001, \eta_{\mathrm{p}}^{2}=0.562$, with faster RTs in the short Tone duration than in the long duration (mean difference $=$ $-9.136 \mathrm{~ms}, \mathrm{SE}=1.553 \mathrm{~ms}, \mathrm{p}<.001$, Cohen's $\mathrm{d}=-0.342$, see Fig. 7, right panel). Note, the main effect of Tone duration here had an opposite direction compared with that in Experiment 3 (in which RTs were quicker in the long than the short tone durations, see Fig. 7, left panel). The main effects of the cue-target interval, $F(1,27)=32.482$, p < $.001, \eta_{\mathrm{p}}^{2}=0.546$, Cue validity, $\mathrm{F}(1,27)=58.187, \mathrm{p}<$ $.001, \eta^{2}=0.683$, and Tone location, $F(1.568,42.349)=$ 97.417, $\mathrm{p}<.001, \eta_{\mathrm{p}}^{2}=0.783$ (see Table 2), were also significant.

There was a significant interaction between Tone duration and Tone location, $F(2,54)=4.725, p=.013, \eta_{p}^{2}=0.149$. Simple effect analyses showed significant RT differences between the short tone duration and the long tone duration when the tones occurred in the middle location (mean difference $=-8.898 \mathrm{~ms}$, SE $=2.437 \mathrm{~ms}, \mathrm{p}=.001$, Cohen's $\mathrm{d}=-0.339$ ) and the late location (mean difference $=-15.976 \mathrm{~ms}, \mathrm{SE}=3.667 \mathrm{~ms}, \mathrm{p}<.001$, Cohen's $d=-0.444)$, but not in the early location $(p=.346)$. 


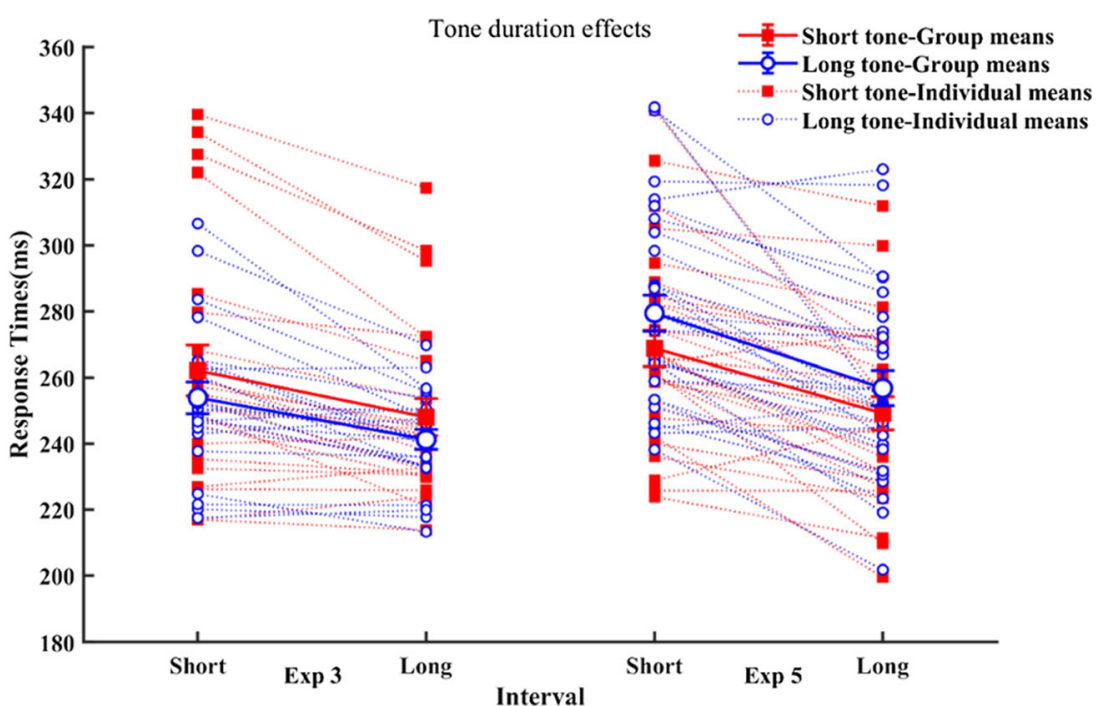

Fig. 7 The Tone duration effects for temporal orienting in Experiment 3 (left) and 5 (right). Individual and group means of response times (RTs) for temporal orienting were shown. RTs were significantly quicker in the long tone durations than the short tone durations in Experiment 3 (left), but showed opposite patterns in Experiment 5 (right)

Cue-target Interval and Cue validity, F $(1,27)=35.059, \mathrm{p}<.001$, $\eta_{p}^{2}=0.565$, see Table 2) was found, similar to Experiment 1a.

was also significant (see Fig. 8, left panel), F(2, 54) = 27.803, p < $.001, \eta_{p}^{2}=0.507$. Further slope analyses (see Table 2) showed that the slope in the short interval (mean $=1.148, \mathrm{SE}=0.470$ ) was significantly larger $(\mathrm{t}(27)=7.66, \mathrm{p}<.001$, Cohen's $\mathrm{d}=$ 0.991 , mean difference $=0.455, \mathrm{SE}=0.594$ ) than that in the long interval (mean $=0.693, \mathrm{SE}=0.430$ ). Thus, the magnitude of the location effect was stronger in the short than the long intervals. Similarly, a temporal orienting effect (a significant interaction of

\section{The influence of temporal orienting on explicit time reproduction}

A $2 \times 2 \times 2 \times 3$ repeated-measures ANOVA with Tone duration (short, long), Cue-target Interval (short, long), Cue validity (Valid, Invalid), and Tone location (early, middle and late)

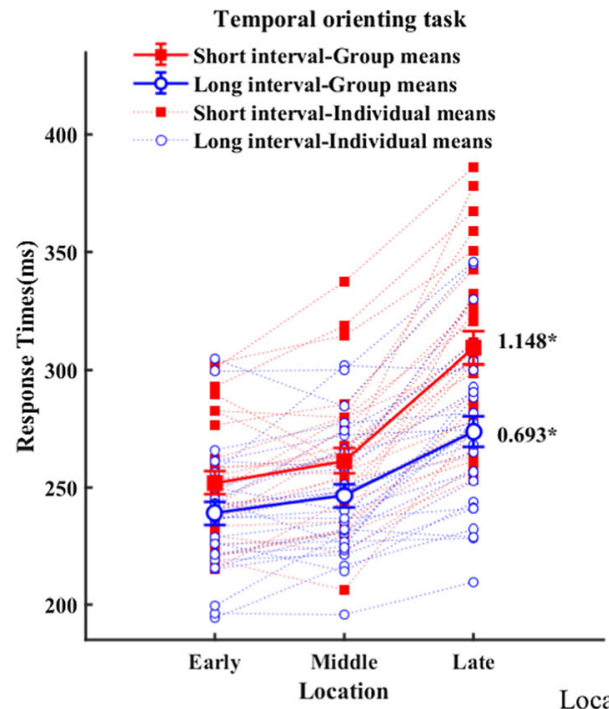

Fig. 8 The location effects measured by response times (RTs) of the target detection (left panel) and by RATIOs of the duration reproduction (right panel) in Experiment 5. RTs of the target detection increased significantly as the locations of the tone onsets closer to the targets for both the short $(2,000 \mathrm{~ms})$ and the long $(3,500 \mathrm{~ms})$ cue-target

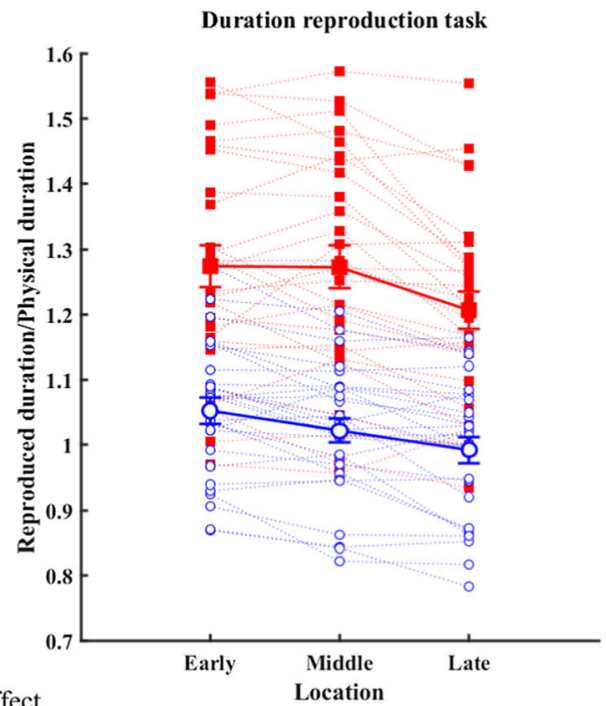

intervals (left panel). The RATIOs of the duration reproduction decreased significantly as the locations of the to-be-encoded tones closer to the targets for both the short and the long intervals (right panel). Other figure formats are similar to Fig. 2 
as factors was conducted on RATIOs of the duration reproduction.

\section{Reproduced-to-physical-duration RATIO}

There was a main effect of Tone duration, $\mathrm{F}(1,27)=154.137$, $\mathrm{p}<.001, \eta_{\mathrm{p}}^{2}=0.851$, indicating over-reproductions for the short than the long tones (mean difference $=0.133, \mathrm{SE}=$ $0.011, p<.001$, Cohen's $d=1.053)$. The main effect of Cue-target Interval was significant, F $(1,27)=134.731, \mathrm{p}<$ $.001, \eta_{p}^{2}=0.833$. Participants over-reproduced the durations (mean difference $=0.228, \mathrm{SE}=0.020, \mathrm{p}<.001$, Cohen's $\mathrm{d}=$ $1.702)$ in the short interval (mean $=1.251, \mathrm{SE}=0.03)$ compared with in the long interval (mean $=1.023, \mathrm{SE}=0.019$ ).

The main effect of Tone location was significant, F (1.39, $37.521)=29.845, \mathrm{p}<.001, \eta_{\mathrm{p}}^{2}=0.525$. There were significant ratio differences between the early and the late tone locations (mean difference $=.064, \mathrm{SE}=.011, \mathrm{p}<.001$, Cohen's d $=0.509$ ), the middle and the late tone locations (mean difference $=.048, \mathrm{SE}=.007, \mathrm{p}<.001$, Cohen's $\mathrm{d}=0.389$ ), and a marginal difference between the early and the middle tone locations $(\mathrm{p}<.088)$. There was also an interaction between Cue-target Interval and Tone location (see Fig. 8, right panel), $F(2,54)=3.308, p=.044, \eta_{p}^{2}=0.109$. Simple effect analyses demonstrated no significant ratio difference between the early and the middle tone locations in the short interval $(\mathrm{p}=.99)$. However, the RATIO differences between all the other location pairs were significant for both short and long intervals (ps $<.05)$.

\section{Discussion}

Two typical effects observed in Experiment 4, i.e., temporal orienting effect and location effect for temporal orienting, were replicated in Experiment 5. The former one indicated that participants in Experiment 5 were carrying out an implicit timing while performing an explicit duration encoding. The latter one reflected that the influence from the explicit timing to the temporal orienting was likely due to a disruption to the temporal-pulse accumulation of the temporal orienting. The effect of Tone duration on temporal orienting was also observed (see Fig. 7, right), which confirmed that besides the interference task difficulties in Experiment 3 (see Fig. 7, left) the degree of temporal-pulse-accumulation overlaps between the dual tasks can also modulate the temporal orienting. The longer the tone duration, the greater the overlap between implicit and explicit timing. The greater overlap produced heavier temporal-pulse-accumulation conflicts and fewer accumulated temporal pulses in the temporal-orienting task (leading to increased RTs). In contrast, such temporal-pulseaccumulation conflicts were absent in Experiment 3 (since the pitch discrimination did not rely on temporal processing and contained no temporal-pulse accumulation). Thus, the direction of the tone duration effect was decided by the difficulty of the non-temporal interference in Experiment 3, leading to an effect opposite to Experiment 5.

Another supporting evidence to a shared temporal-pulse accumulation between the implicit and explicit timing arose from the fact that there was a RATIO-based location effect for the tone reproduction (see Fig. 8, right; i.e., a location effect for the interference task), echoing to the RT-based location effect for the temporal orienting (see Fig. 8, left). This RATIO-based location effect demonstrated that the perceived duration of the tone (proportional to the RATIO value) actually decreased as the location level increased, suggesting an increasing loss of temporal pulses from the early to the late location levels during the tone duration encoding. This is consistent with a previous study (Cicchini \& Morrone, 2009) demonstrating that attentional diversion during the temporal encoding of an event can lead to a decrease in its perceived duration.

The fact that the location effect for the interference task only emerged in Experiment 5 but not in Experiment 4 ruled out the possibility of "a simple interference hypothesis" that claimed that the location effect for temporal orienting was induced simply by processing interference between the temporal-orienting task and the interference task per se. Also, the results of Experiments 4 and 5 ruled out the possibility of "a foreperiod effect-based hypothesis" that claimed that the location effect for temporal orienting was only a byproduct of the resource reallocation on the interference stimuli at different location levels. According to this hypothesis, more attentional resource should be allocated to the tones at later location levels. Thus, we would expect to observe a performance increase of the pitch discrimination at later location levels in Experient 4. However, the absence of locational modulation on the pitch discrimination suggested that the location effect may not originate from the tone-related FP effect. Furthermore, as indicated by previous studies (Grondin \& Rammsayer, 2003; Los \& Horoufchin, 2011), a signature phenomenon for the FP effect when an explicit timing was required for the $\mathrm{S} 2$ stimuli was that the perceived duration of the S2-defined interval increased with the FPs. If the FP effect did occur for the tones, we would expect to observe an increased duration reproduction at later location levels in Experiment 5. Clearly, Experiments 4 and 5 provided no support to the "foreperiod effect-based hypothesis." Instead, the results of Experiment 5, particularly the two-way location effects and the tone-duration effect for temporal orienting, along with the results of Experiment 4 provided evidence that the internal representation of temporal orienting was similar to those of explicit duration reproduction.

The mutual influence between the implicit temporal orienting and the explicit duration reproduction was consistent with a previous study (McAuley \& Jones, 2003) that demonstrated, by means of a combined qualitative analyses and 
quantitative modeling, that implicit temporal structures hidden in a rhythmic context can be extracted automatically and influence subsequent timing judgment between a standard and a comparison interval. The occurrence of time distortions depended on whether the ending time of the standard interval was consistent (i.e., their "on-time" condition) with the expected time predicted by the rhythmic context, rather than whether its absolute temporal distance from the preceding rhythmic context was near (i.e., their "advancing" condition, supposedly having greater interference as predicted by the foregoing "simple interference hypothesis") or far (i.e., their "delaying" condition, supposedly having less interference). The expectations along time, extracted from an implicit timing either automatically (as in McAuley \& Jones, 2003) or by controlled attention (as in our Experiment 5), can modulate the final "count" of an explicit timing, in ways beyond the simple interference and whether the implicit and the explicit timing were arranged in a close sequence (as in McAuley \& Jones, 2003) or in a nested overlap (as in our Experiment 5). These facts (from these two studies) implied that the internal representations of implicit and explicit timing are unlikely independent from each other.

\section{General discussion}

Studies on implicit timing demonstrated that we can improve our accuracy and speed of processing to upcoming information (Breska \& Deouell, 2014) by narrowing down attention into a future moment when a key stimulus is likely to occur. This reminded us about how attention was able to be focused into a three-dimensional space in the case of spatial selective attention (Posner, Snyder, \& Davidson, 1980). For that reason, implicit timing was dubbed as the "the fourth dimension in attention" (Nobre \& van Ede, 2018). The present study aimed to explore the mechanisms of the internal temporal representations during temporal orienting. This issue has potential theoretical contribution to the existing debates on the similarities and differences between explicit and implicit timing.

In six experiments, a dual-task approach was used to test the nature of the processes in temporal orienting. Our results demonstrated that temporal orienting was modulated by properties of the interference tasks, including temporal locations and processing demands of the interference stimuli. Furthermore, the modulating effects from two different interference tasks (i.e., the one in Experiment 5, which contained a temporal-pulse-accumulation component and the one in Experiment 4, which did not) were investigated and compared. Temporal-pulse-accumulation conflicts were manifested by two-way location effects (one for temporal orienting and the other for the interference task) in Experiment 5, but not in Experiment 4 in which a one-way location effect (for temporal orienting only) was observed. A tone duration effect for temporal orienting (slower RTs with longer tone durations) was also revealed in Experiment 5, suggesting greater temporalpulse-accumulation conflicts for conditions with longer tone durations. Taken together, these results provided evidence that implicit timing, such as temporal orienting, possessed mechanisms of temporal-pulse accumulation and attentional gating, i.e., similar to explicit timing (Fortin \& Tremblay, 2006).

\section{An explanatory model for the location effect for temporal orienting}

Next, we proposed a temporal-pulse accumulation and attentional gating-based account to explain the location effect for temporal orienting and its characteristics, by borrowing similar logics from Fortin and Tremblay (2006)'s theoretic framework for explicit timing. The black slash curve a in Fig. 9 represented the temporal-pulse accumulation during a single task of temporal orienting. There was no specific perturbation in this condition, thus the speed of temporal-pulse accumulation (represented as slope $\mathrm{S}_{0}$ in Fig. 9) was constant. However, in conditions when there was an additional interference task (the red slash curve b or the yellow slash curve c in Fig. 9), attention need to be shared between the temporal orienting and the online monitoring to the occurrence of the interference stimuli (which was earlier in $b$ than that in c). The latter process required controlled attention and thus diverted attention away from temporal orienting. This triggered a perturbation on the temporal-pulse accumulation of temporal orienting and rendered the speed of temporal-pulse accumulation, i.e., the slopes of curve $b$ and curve $c\left(S_{1}\right.$ in Fig. 9), reduced, relative to

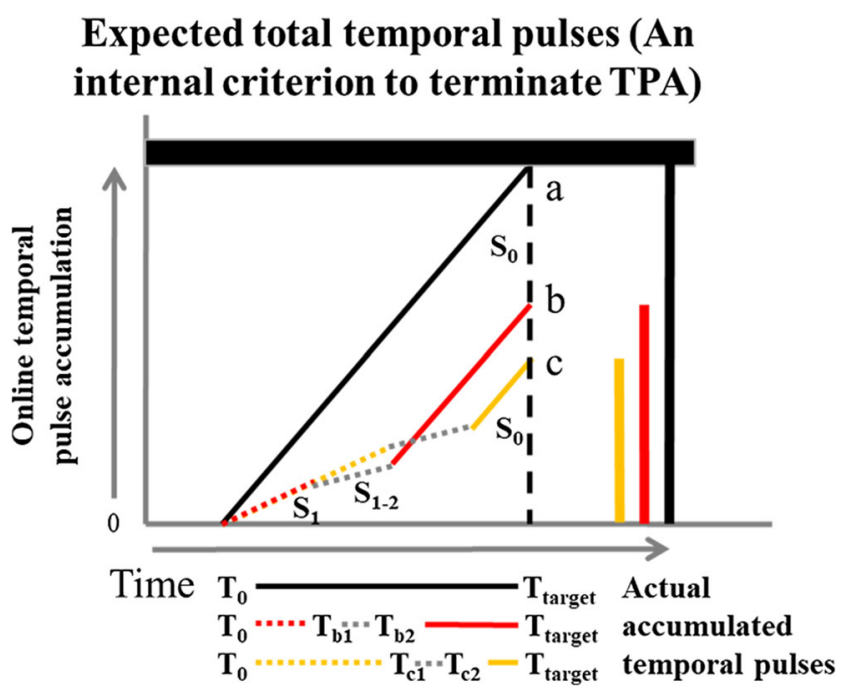

Fig. 9 Schematic diagram of the location effect for temporal orienting, adapted from the model for the location effect for explicit timing (Fortin \& Tremblay, 2006). 'S' and 'T' stand for 'Slope' and 'Time', respectively 
the slope of the curve a (the upper $S_{0}$ in Fig. 9) during the time window from the start of the temporal-pulse accumulation in temporal orienting ( $T_{0}$ in Fig. 9 ) to the end times of the online monitoring (i.e., the occurrence times of the interference stimuli, $\mathrm{T}_{\mathrm{b} 1}$ for curve $\mathrm{b}$ and $\mathrm{T}_{\mathrm{c} 1}$ for curve $\mathrm{c}$ in Fig. 9, respectively). The slow-down of the accumulation process would cause a relative loss of temporal information. Only when the interference task was completed and disengaged from the temporalorienting task completely $\left(\mathrm{T}_{\mathrm{b} 2}\right.$ for curve $\mathrm{b}$ and $\mathrm{T}_{\mathrm{c} 2}$ for curve $\mathrm{c}$ in Fig. 9, respectively), the attention sharing stops and the slopes of pulse accumulation of the curve $b$ and c could be restored into a level (the lower $S_{0}$ in Fig. 9) as equal as the slope of the curve a. The recovery of temporal-pulseaccumulation slope from $S_{1}$ to $S_{0}$ took about equal time for curve $\mathrm{b}\left(\mathrm{T}_{\mathrm{b} 2-\mathrm{b} 1}=\mathrm{T}_{\mathrm{b} 2}-\mathrm{T}_{\mathrm{b} 1}\right)$ and curve $\mathrm{c}\left(\mathrm{T}_{\mathrm{c} 2-\mathrm{c} 1}=\mathrm{T}_{\mathrm{c} 2}-\mathrm{T}_{\mathrm{c} 1}\right)$. Note that in order to simplify the model, we used a constant slope $\left(S_{1-2}\right.$ in Fig. 9$)$ to describe the slope recovery from $T_{b 1}$ to $T_{b 2}$ and that from $T_{c 1}$ to $T_{c 2}$. This recovery process was not necessarily linear. However, relative to the difference of the location levels between curve $\mathrm{b}$ and c, i.e., $\left(\mathrm{T}_{\mathrm{c} 1}-\mathrm{T}_{0}\right)-\left(\mathrm{T}_{\mathrm{b} 1}-\mathrm{T}_{0}\right)$ $=T_{c 1}-T_{b 1}$, the difference of the slope recovery time between curve $b$ and c, i.e., $T_{b 2-b 1}-T_{c 2-c 1}$, was negligible. In that sense, the dominant reason of the location effect came from the stage of the speed slow-down with a slope of $S_{1}$, rather than the stage of the slope recovery from $S_{1}$ to $S_{0}$. Thus, neither the linear or nonlinear definition of the slope recovery was dominant in the explanation of the location effect for temporal orienting. More specifically, the final accumulated temporal pulses (which decided the temporal orienting performance, i.e., $\mathrm{RTs})$ for curve $\mathrm{a}\left(\mathrm{TP}_{\mathrm{a}}\right), \mathrm{b}\left(\mathrm{TP}_{\mathrm{b}}\right)$ and $\mathrm{c}\left(\mathrm{TP}_{\mathrm{c}}\right)$ were:

$$
\begin{aligned}
\mathrm{TP}_{\mathrm{a}} & =\left(\mathrm{T}_{\mathrm{b} 1}-\mathrm{T}_{0}\right) * \mathrm{~S}_{0}+\left(\mathrm{T}_{\mathrm{b} 2}-\mathrm{T}_{\mathrm{b} 1}\right) * \mathrm{~S}_{0}+\left(\mathrm{T}_{\text {target }}-\mathrm{T}_{\mathrm{b} 2}\right) * \mathrm{~S}_{0} \\
& =\left(\mathrm{T}_{\text {target }}-\mathrm{T}_{0}\right) * \mathrm{~S}_{0}
\end{aligned}
$$

$$
\begin{aligned}
\mathrm{TP}_{\mathrm{b}}= & \left(\mathrm{T}_{\mathrm{b} 1}-\mathrm{T}_{0}\right) * \mathrm{~S}_{1}+\left(\mathrm{T}_{\mathrm{b} 2}-\mathrm{T}_{\mathrm{b} 1}\right) * \mathrm{~S}_{1-2} \\
& +\left(\mathrm{T}_{\text {target }}-\mathrm{T}_{\mathrm{b} 2}\right) * \mathrm{~S}_{0} \\
\mathrm{TP}_{\mathrm{c}}= & \left(\mathrm{T}_{\mathrm{c} 1}-\mathrm{T}_{0}\right) * \mathrm{~S}_{1}+\left(\mathrm{T}_{\mathrm{c} 2}-\mathrm{T}_{\mathrm{c} 1}\right) * \mathrm{~S}_{1-2} \\
& +\left(\mathrm{T}_{\text {target }}-\mathrm{T}_{\mathrm{c} 2}\right) * \mathrm{~S}_{0}
\end{aligned}
$$

Since $\mathrm{T}_{\mathrm{b} 2-\mathrm{b} 1} \approx \mathrm{T}_{\mathrm{c} 2-\mathrm{c} 1}$, the difference between $\mathrm{TP}_{\mathrm{b}}$ and $\mathrm{TP}_{\mathrm{c}}$ was:

$$
\begin{aligned}
\mathrm{TP}_{\mathrm{b}}-\mathrm{TP}_{\mathrm{c}}= & \left(\mathrm{T}_{\mathrm{b} 1}-\mathrm{TP}_{\mathrm{c} 1}\right)^{*} \mathrm{~S}_{1}+\left(\mathrm{T}_{\mathrm{c} 2}-\mathrm{TP}_{\mathrm{b} 2}\right)^{*} \mathrm{~S} 0=\left(\mathrm{T}_{\mathrm{b} 1}-\mathrm{TP}_{\mathrm{c} 1}\right)^{*} \mathrm{~S}_{1} \\
& +\left(\left(\mathrm{T}_{\mathrm{c} 1}+\mathrm{T}_{\mathrm{c} 2}-\mathrm{c} 1\right)-\left(\mathrm{T}_{\mathrm{b} 1}+\mathrm{T}_{\mathrm{b} 2}-\mathrm{b}_{1}\right)\right)^{*} \mathrm{~S}_{0} \\
= & \left(\mathrm{T}_{\mathrm{b} 1}-\mathrm{TP}_{\mathrm{c} 1}\right)^{*} \mathrm{~S}_{1}+\left(\mathrm{T}_{\mathrm{c} 1}-\mathrm{TP}_{\mathrm{b} 1}\right)^{*} \mathrm{~S}_{0}=\left(\mathrm{T}_{\mathrm{c} 1}-\mathrm{T}_{\mathrm{b} 1}\right)^{*}\left(\mathrm{~S}_{0}-\mathrm{S}_{1}\right)
\end{aligned}
$$

The above calculations suggest that $\mathrm{TP}_{\mathrm{a}}>\mathrm{TP}_{\mathrm{b}}>\mathrm{TP}_{\mathrm{c}}$. Analogous to Fortin and Tremblay (2006)'s theoretic framework for explicit timing, the temporal orienting should be the most accurate only if the actual number of accumulated temporal pulses was as close as possible to the expected number of total temporal pulses, i.e., an internal criterion to terminate temporal-pulse accumulation. Thus, the curve $\mathrm{c}$ had the greatest difference of the temporal pulses away from the internal criterion, resulting in the least accurate temporal orienting and the slowest RT, compared with the curve $b$ and a.

Since the magnitude of the location effect $\left(\mathrm{LE}_{\text {slope }}\right)$ can be characterized as a slope, i.e., difference of the accumulated temporal pulses between two location levels $\left(\mathrm{TP}_{\mathrm{b}}-\mathrm{TP}_{\mathrm{c}}\right) \mathrm{di}$ vided by Time difference between the two location levels $\left(\mathrm{T}_{\mathrm{c} 1}\right.$ $-\mathrm{T}_{\mathrm{b} 1}$ ), then:

$\mathrm{LE}_{\text {slope }}=\left(\mathrm{TP}_{\mathrm{b}}-\mathrm{TP}_{\mathrm{c}}\right) /\left(\mathrm{T}_{\mathrm{c} 1}-\mathrm{T}_{\mathrm{b} 1}\right)=\mathrm{S}_{0}-\mathrm{S}_{1}$

Thus, the magnitude of the location effect for temporal orienting was solely decided by the difference between a single-task speed of temporal-pulse accumulation $\left(\mathrm{S}_{0}\right)$ and a reduced speed of temporal-pulse accumulation $\left(\mathrm{S}_{1}\right)$ due to the monitoring to the interference stimuli. This conclusion was consistent with the task difficulty-sensitivity found in Experiment 3 in that the magnitude of $\mathrm{S}_{1}$ should be influenced by expectations on how difficult it would be to detect the upcoming interference stimuli. For the shorter and more difficult tones in Experiment 3, more attentional resources were required to monitor the tones' occurrence. Thus, less attention was allocated to the temporal orienting, leading to a reduced $\mathrm{S}_{1}$ and an increased location effect, relative to conditions with easier interference stimuli. The above explanation is also consistent with our finding that the task difficulty-sensitivity was transient and modulated by encountering/learning experience (i.e., the tone order effect in Experiment 3). It is conceivable that the expectation to the difficulty levels of the interference stimuli and the resultant $S_{1}$ could be dynamically adjusted by whether easy or difficult interference task was encountered and performed first. Similar task difficulty-sensitivities and tone order effects were also observed in explicit timing (Casini \& Macar, 1997; Fortin, 2003).

\section{Architecture and constraints of the human timing system}

Experiment 5 demonstrated temporal-pulse-accumulation conflicts between implicit and explicit timing. Similar conflicts of temporal processing, though across multiple explicit timings rather than between an explicit and an implicit timing (as in our Experiment 5), had been observed in several studies (Ayhan et al., 2012; Cheng et al., 2014; van Rijn \& Taatgen, 2008) when multiple, temporally overlapping durations required concurrent processing.

The temporal-pulse-accumulation conflicts in Experiment 5 might shed some light on our understanding to the 
architecture and constraints of the human timing system. In order to account for simultaneous timing on two overlapping intervals, previous literature on explicit timing had proposed three scalar-expectancy-theory (SET, Gibbon, 1977)-based models to elaborate potential computational implementations of a temporal-pulse accumulation and attentional gating-based timing system. The first model (Bryce \& Bratzke, 2016; Bryce, Seifried-Dübon, \& Bratzke, 2015) suggested that we used a structure consisting of a single pacemaker and a single accumulator (denoted as SPSA model) to estimate two overlapping intervals (e.g., a short one nested in a long one) simultaneously. According to this model, participants should segment the two overlapping intervals into three non-overlapping and overlapping segments first, and time these segments separately before summing them to estimate each interval. Critically, the summation was free from the influence of dual-task costs (Bryce \& Bratzke, 2016). On the basis that temporal orienting also involves temporal-pulse accumulation and that the cue-target interval and the tone-occupied interval in our Experiment 5 were akin to the long and the short intervals in the SPSA model, respectively, the perceived duration of the tones in Experiment 5 should be unaffected by the SOAs between the two intervals (equivalent to the location levels in the present study) since the estimation of the short interval did not rely on the summation of the three segments. Clearly, this prediction was not fulfilled by the RATIO-based location effect in Experiment 5. In contrast to the SPSA model, the other two pacemaker-accumulator models emphasized that multiple accumulators (MA), each for one interval processing, were involved instead. A single pacemaker (SP), shared between processing of the long and short intervals (Rousseau \& Rousseau, 1996), was adopted in the second model (denoted as SPMA model), whereas multiple pacemakers (MP), each for one interval processing (Crystal, 2003), were taken in the third model (denoted as MPMA model). Both the SPMA and MPMA models regarded dualtask costs as the dominant factors affecting the estimates of both intervals and predicted that both perceived intervals would decrease with increasing location levels (Bryce \& Bratzke, 2016), a prediction consistent with our Experiment 5. Thus, we posit that separate accumulators, one for implicit timing and the other for explicit timing, were underlying a dual-task paradigm involving implicit and explicit timing simultaneously.

It is also noteworthy that although the SPMA or the MPMA models provided the most parsimonious explanation to the current results, there are other theoretical propositions (for a review, see Lewis \& Miall, 2006), beyond pacemakeraccumulator models, for explaining the ability to keep track of time. For instance, Staddon and Higa (1999) proposed that decay of activation in memory, rather than any type of pacemaker, can provide enough information to track the passage of time. Thus, an interesting question is: would it be possible that memory decay-related effects, including representation maintenance and decay in a reference memory as a measure of previously elapsed time, or subsequent discrimination on the strengths of memory traces (Roberts, 2002), produce the observed location effects here? Experiment 5 deliberately controlled the potential effect of memory decay by keeping the temporal distance between the tone offset and the start of the reproduction phase constant. Yet, a RATIO-based location effect was observed, suggesting that the memory process-related timing account (Staddon \& Higa, 1999), different from those based on SET, cannot provide dominant explanation to the temporal processing in the present study.

It is interesting to ask further what is the signature difference of temporal processing between a temporalorienting task and an explicit-timing task. In line with many previous studies (Merchant et al., 2008; Spencer \& Zelaznik, 2003), we demonstrated that a temporal-pulse accumulation and attentional gating-based mechanism was activated in a temporal-orienting task, similar to that in an explicit duration reproduction. This implied the possibility that a common set of cognitive modules, such as those underlying the attentional-gate model (Zakay \& Block, 1997), takes part in temporal processing, regardless of whether it is explicit or implicit. In contrast, a complete implementation of any explicit timing might rely on an additional component of cognitive processing. Specifically, the internal temporal representation, such as the final count of the accumulated temporal pulses in the accumulator, is likely to be accessed and operated at the conscious level during its comparison with a previously stored reference in working memory.

Here, we tentatively proposed that the signature difference of temporal processing between implicit and explicit timing relies on this additional processing component, i.e., the conscious accessing and operating on internal temporal representations in the pacemaker-accumulator timing system, which is absent in temporal orienting but present in explicit duration reproduction. This account helps to reconcile the seemingly conflicting results in the current literature. On one hand, the usage of the same temporal representations in a common pacemaker-accumulator timing system leads to adherence to the Weber's law and the Proportional timing by both implicit and explicit timing (Piras \& Coull, 2011). On the other hand, the extra cognitive component in explicit timing (over and above implicit timing) can explain the different neural substrates (Coull et al., 2013) and developmental dissociations between these two types of timing (Droit-Volet \& Coull, 2016).

The above proposal is consistent with recent literature. For example, the conscious monitoring of temporal representations in an explicit-timing task relied heavily on the developmental 
increase in executive control functions (Cleeremans \& Jiménez, 2002; Reber, 1992), which do not reach a full maturity until an age of at least 8-12 years (Karmiloff-Smith, 1994). This can explain why the variability of temporal performance decreased as a function of age in explicit timing, whereas it remained constant for 5-year-olds and adults and was unrelated to development of general cognitive capacity in implicit timing (Droit-Volet \& Coull, 2016). Also, the cortical lateralization for implicit and explicit timing (left/right hemispheric specificity for implicit/explicit timing, Coull et al., 2013) likely reflects different ways in which temporal representations were ultimately used. Taken together, the common cognitive components between implicit and explicit timing, i.e., the pacemakeraccumulator timing modules, seem to be more primitive and emerge earlier ontogenetically than the conscious monitoring of temporal information necessary for explicit timing.

It is noteworthy that temporal orienting is only one form of implicit timing (for a review, see Coull \& Nobre, 2008). It is of course an open question for future research to ask whether similar location effect also occurs in situations of other types of implicit timing beyond temporal orienting, such as the implicit timing due to rhythmic stimulus features (McAuley \& Jones, 2003) or motor- rather than perception-based implicit timing (Zelaznik et al., 2002).

\section{Conclusion}

We showed that a temporal-orienting task can be undermined by a concurrent interference task, leading to a location effect for temporal orienting. The magnitude of the location effect was sensitive to whether automatic or controlled attentional shifts were involved and the difficulties of the interference stimuli. When an explicit tone duration reproduction was taken as the interference task, a tone duration effect and two-way location effects were observed, respectively. The above results provided strong evidence that implicit timing (e.g., temporal orienting) and explicit timing might share common mechanisms of internal temporal representations in terms of temporal-pulse accumulation and attentional gating.

Supplementary Information The online version contains supplementary material available at https://doi.org/10.3758/s13414-020-02176-y.

Open Practices Statement None of the experiments reported in this article were formally preregistered. The data for all experiments are available as Electronic Supplementary Material to this article.

Funding This work was made possible by grants from the National Natural Science Foundation of China (31671122 and 31500869) and the Fundamental Research Funds for the Central Universities, China (CCNU19TS039, CCNU19TS075, CNU18TS037 and CCNU19TD019).

\section{Compliance with ethical standards}

Conflicts of interest The authors declare that they have no conflicts of interest.

Ethical approval All procedures performed in studies involving human participants were in accordance with the ethical standards of the institutional and/or national research committee, the American Psychological Association (APA) standards and with the 1964 Helsinki declaration and its later amendments or comparable ethical standards.

\section{References}

Ameqrane, I., Pouget, P., Wattiez, N., Carpenter, R., \& Missal, M. (2014). Implicit and Explicit Timing in Oculomotor Control. PLoS ONE, 9(4), e93958.

Artieda, J., Pastor, M. A., Lacruz, F., \& Obeso, J. A. (1992). Temporal discrimination is abnormal in Parkinson's disease. Brain, 115(1), 199-210.

Ayhan, I., Revina, Y., Bruno, A., \& Johnston, A. (2012). Duration judgments over multiple elements. Frontiers in Psychology, 3(459).

Bertelson, P., \& Tisseyre, F. (1968). The time-course of preparation with regular and irregular foreperiods. Quaterly Journal of Experimental Psychology, 20, 297-300.

Breska, A., \& Deouell, L. Y. (2014). Automatic Bias of Temporal Expectations following Temporally Regular Input Independently of High-level Temporal Expectation. Journal of Cognitive Neuroscience, 26(7), 1555-1571.

Bryce, D., \& Bratzke, D. (2016). Multiple timing of nested intervals: Further evidence for a weighted sum of segments account. Psychonomic Bulletin \& Review, 23(1), 317-323.

Bryce, D., Seifried-Dübon, T., \& Bratzke, D. (2015). How are overlapping time intervals perceived? Evidence for a weighted sum of segments model. Acta Psychologica, 156, 83-95.

Bueti, D., Walsh, V., Frith, C., \& Rees, G. (2008). Different brain circuits underlie motor and perceptual representations of temporal intervals. Journal of Cognitive Neuroscience, 20(2), 204-214.

Buffardi, L. (1971). Factors affecting the filled-duration illusion in the auditory, tactual, and visual modalities. Perception \& Psychophysics, 10, 292-294.

Casini, L., \& Macar, F. (1997). Effects of attention manipulation on judgments of duration and of intensity in the visual modality. Memory \& Cognition, 25(6), 812-818.

Casini, L., Ramdani-Beauvir, C., Burle, B., \& Vidal, F. (2013). How does one night of sleep deprivation affect the internal clock? Neuropsychologia, 51(2), 275-283.

Champagne, J., \& Fortin, C. (2008). Attention sharing during timing: Modulation by processing demands of an expected stimulus. Perception \& Psychophysics, 70(4), 630-639.

Cheng, X., Yang, Q., Han, Y., Ding, X., \& Fan, Z. (2014). Capacity Limit of Simultaneous Temporal Processing: How Many Concurrent 'Clocks' in Vision? PLoS ONE, 9(3), e91797.

Cicchini, G. M., \& Morrone, M. C. (2009). Shifts in spatial attention affect the perceived duration of events. Journal of Vision, 9(1): 113.

Cleeremans, A., \& Jiménez, L. (2002). Implicit learning and consciousness: A graded, dynamic perspective. In R. M. French \& A. Cleeremans (Eds.), Implicit learning and consciousness (pp. 140). Hove, UK: Psychology Press. 
Cohen, J. (1988). Statistical power analysis for the behavioral sciences. New York: Academic Press.

Coull, J. T., Cheng, R., \& Meck, W. H. (2011). Neuroanatomical and neurochemical substrates of timing. Neuropsychopharmacology, $36(1), 3-25$.

Coull, J. T., Davranche, K., Nazarian, B., \& Vidal, F. (2013). Functional anatomy of timing differs for production versus prediction of time intervals. Neuropsychologia, 51(2), 309-319.

Coull, J. T., \& Nobre, A. C. (1998). Where and when to pay attention: the neural systems for directing attention to spatial locations and to time intervals as revealed by both PET and fMRI. The Journal of Neuroscience, 18(18), 7426-7435.

Coull, J. T., \& Nobre, A. C. (2008). Dissociating explicit timing from temporal expectation with fMRI. Current Opinion in Neurobiology, 18(2), 137-144.

Creelman, C. D. (1962). Human discrimination of auditory duration. Journal of the Acoustical Society of America, 34, 582-593.

Crystal, J. D. (2003). Nonlinearities in sensitivity to time: Implications for oscillatorbased representations of interval and circadian clocks. In W. H. Meck (Ed.), Functional and neural mechanisms of interval timing (pp. 61-77). Boca Raton, FL: CRC Press.

Droit-Volet, S., \& Coull, J. T. (2016). Distinct developmental trajectories for explicit and implicit timing. Journal of experimental child psychology, 150, 141-154.

Fan, Z., Singh, K. D., Muthukumaraswamy, S. D., Sigman, M., Dehaene, S., \& Shapiro, K. (2012). The cost of serially chaining two cognitive operations. Psychological Research, 76, 566-578.

Faul, F., Erdfelder, E., \& Lang, A.-G. (2007). G Power 3: A flexible statistical power analysis program for the social, behavioral, and biomedical sciencesBuchner, A. Behavior Research Methods, 39, 175-191.

Fautrelle, L., Mareschal, D., French, R., Addyman, D., \& Thomas, E. (2015). Motor activity improves temporal expectancy. PLoS ONE, 10(3), e 0119187.

Fortin, C. (2003). Attentional Time-Sharing in Interval Timing. In W. H. Meck (Ed.), Functional and Neural Mechanisms of Interval Timing (pp. 235-260): CRC Press.

Fortin, C., Bédard, M.-C., \& Champagne, J. (2005). Timing during interruptions in timing. Journal of Experimental Psychology: Human Perception \& Performance, 31(2), 276-288.

Fortin, C., \& Massé, N. (2000). Expecting a break in time estimation: attentional time- sharing without concurrent processing. Journal of Experimental Psychology: Human Perception \& Performance, 26(6), 1788-1796.

Fortin, C., \& Tremblay, S. (2006). Interrupting timing in interval production and discrimination: similarities and differences. Behavioural Processes, 71(2), 336-343.

Gaudreault, R., \& Fortin, C. (2013). To count or not to count: the effect of instructions on expecting a break in timing. Attention, Perception, \& Psychophysics, 75(3), 588-602.

Gaudreault, R., Fortin, F., \& Macar, F. (2010). Contrasting effects of interference and of breaks in interval timing. Acta Psychologica, 133(1), 3-16.

Gibbon, J. (1977). Scalar expectancy theory and Weber's law in animal timing. Psychological Review, 84(3), 279-325.

Gibbon, J., Church, R. M., \& Meck, W. H. (1984). Scalar timing in memory. In J. Gibbon \& L. Allan (Eds.), Timing and time perception (pp. 52-77). NewYork: NewYork Academy of Sciences.

Grondin, S. (2010). Timing and time perception: A review of recent behavioral and neuroscience findings and theoretical directions. Attention, Perception, \& Psychophysics, 72(3), 561-582.
Grondin, S., \& Rammsayer, T. (2003). Variable foreperiods and temporal discrimination. The quarterly journal of experimental psychology, 56A, 731-765.

Haggard, P., Clark, S., \& Kalogeras, J. (2002). Voluntary action and conscious awareness. Nature Neuroscience, 5(4), 382-385.

Karmiloff-Smith, A. (1994). Pécis of beyond modularity: A developmental perspective on cognitive science. Behavioral and Brain Sciences, 17, 693-745.

Kline, R. B. (2004). Beyond significance testing. Washington, DC: American Psychological Association.

Lewis, P. A., \& Miall, R. C. (2006). Remembering the time: a continuous clock. Trends in Cognitive Science, 10(9), 401-406.

Los, S. A., \& Horoufchin, H. (2011). Dissociative patterns of foreperiod effects in temporal discrimination and reaction time tasks. The quarterly journal of experimental psychology, 64(5), 1009-1020.

Macar, F. (2002). Expectancy, controlled attention and automatic attention in prospective temporal judgments. Acta Psychologica, 111(2), 243-262.

Macar, F., Grondin, S., \& Casini, L. (1994). Controlled attention sharing influences time estimation. Memory \& Cognition, 22, 673-686.

McAuley, J. D., \& Jones, M. R. (2003). Modeling Effects of Rhythmic Context on Perceived Duration: A Comparison of Interval and Entrainment Approaches to Short-Interval Timing. Journal of Experimental Psychology: Human Perception \& Performance, 29(6), 1102-1125.

Meck, W. H. (2005). Neuropsychology of timing and time perception. Brain and Cognition, 58(1), 1-8.

Merchant, H., Zarco, W., Bartolo, R., \& Prado, L. (2008). The context of temporal processing is represented in the multidimensional relationships between timing tasks. PLoS ONE, 3(9), e3169.

Meyer, D. E., \& Kieras, D. E. (1997). A computational theory of executive cognitive processes and multiple-task performance: Part 1. Basic mechanisms. Psychological Review, 104, 3-65.

Niemi, P., \& Näätänen, R. (1981). Foreperiod and simple reaction time. Psychological Bulletin, 89, 133-162.

Nobre, A. C., \& Rohenkohl, G. (2014). Time for the fourth dimension in attention. In A. C. Nobre \& S. Kastner (Eds.), The Oxford handbook of attention. Oxford, UK: Oxford University Press.

Nobre, A. C., \& van Ede, F. (2018). Anticipated moments: temporal structure in attention. Nature Reviews Neuroscience, 19, 34-48.

Pashler, H. (1984). Processing stages in overlapping tasks: evidence for a central bottleneck. Journal of Experimental Psychology: Human Perception \& Performance, 10, 358-377.

Pashler, H. (1994). Dual-task interference in simple tasks: data and theory. Psychological Bulletin, 116, 220-244.

Piras, F., \& Coull, J. T. (2011). Implicit, predictive timing draws upon the same scalar representation of time as explicit timing. PLoS ONE, 6(3), e18203.

Posner, M. I., Snyder, C. R., \& Davidson, B. J. (1980). Attention and the detection of signals. Journal of Experimental Psychology: General, 109(2), 160-174.

Praamstra, P., Kourtis, D., Kwok, H. F., \& Oostenveld, R. (2006). Neurophysiology of implicit timing in serial choice reaction-time performance. The Journal of Neuroscience, 26(20), 5448-5455.

Reber, A. S. (1992). The cognitive unconscious: An evolutionary perspective. Consciousness and Cognition, 1, 93-113.

Roberts, W. A. (2002). Are Animals Stuck in Time? Psychological Bulletin, 128(3), 473-489.

Rousseau, L., \& Rousseau, R. (1996). Stop-reaction time and the internal clock. Perception \& Psychophysics, 58(3), 434-448.

Schwartze, M., Rothermich, K., \& Kotz, S. A. (2012). Functional dissociation of pre-SMA and SMA-proper in temporal processing. NeuroImage, 60(1), 290-298. 
Spencer, R., \& Zelaznik, H. N. (2003). Weber (slope) analyses of timing variability in tapping and drawing tasks. Journal of Motor Behavior, 35(4), 371-381.

Staddon, J. E. R., \& Higa, J. J. (1999). Time and memory: Towards a pacemaker-free theory of interval timing. Journal of the Experimental Analysis of Behavior, 71(2), 215-251.

Treisman, M. (1963). Temporal discrimination and the indifference interval: implications for a model of the 'internal clock'. Psychological Monographs: : General and Applied, 77(13), 1-31.

van Rijn, H., \& Taatgen, N. A. (2008). Timing of multiple overlapping intervals: How many clocks do we have? Acta Psychologica, 129, 365-375.
Woodrow, H. (1914). The measurement of attention. Psychological Monographs, 17, 1-158.

Zakay, D., \& Block, R. A. (1997). Temporal cognition. Current Directions in Psychological Science, 12-16.

Zelaznik, H. N., Spencer, R., \& Ivry, R. B. (2002). Dissociation of explicit and implicit timing in repetitive tapping and drawing movements. Journal of Experiment Psychology: Human Perception \& Performance, 28, 575-588.

Publisher's note Springer Nature remains neutral with regard to jurisdictional claims in published maps and institutional affiliations. 